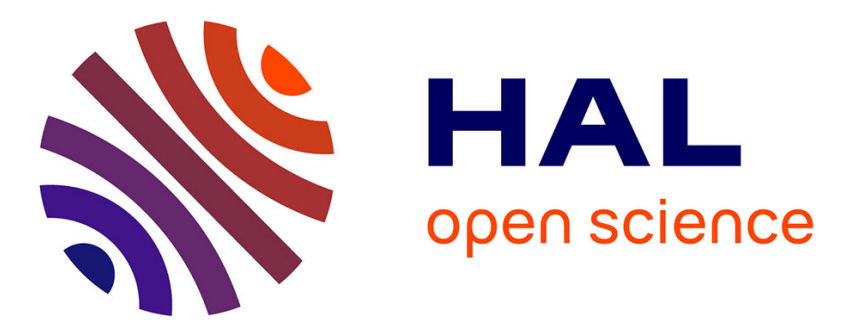

\title{
Zero field splitting of the chalcogen diatomics using relativistic correlated wave-function methods.
}

Jean-Baptiste Rota, Stefan Knecht, Timo Fleig, Dmitry Ganyushin, Trond

Saue, Frank Neese, Hélène Bolvin

\section{- To cite this version:}

Jean-Baptiste Rota, Stefan Knecht, Timo Fleig, Dmitry Ganyushin, Trond Saue, et al.. Zero field splitting of the chalcogen diatomics using relativistic correlated wave-function methods.. Journal of Chemical Physics, 2011, 135 (11), pp.114106. 10.1063/1.3636084 . hal-00760776

\section{HAL Id: hal-00760776 https://hal.science/hal-00760776}

Submitted on 21 Jan 2020

HAL is a multi-disciplinary open access archive for the deposit and dissemination of scientific research documents, whether they are published or not. The documents may come from teaching and research institutions in France or abroad, or from public or private research centers.
L'archive ouverte pluridisciplinaire HAL, est destinée au dépôt et à la diffusion de documents scientifiques de niveau recherche, publiés ou non, émanant des établissements d'enseignement et de recherche français ou étrangers, des laboratoires publics ou privés. 


\section{Zero field splitting of the chalcogen diatomics using relativistic correlated wave- function methods}

Cite as: J. Chem. Phys. 135, 114106 (2011); https://doi.org/10.1063/1.3636084

Submitted: 15 June 2011 . Accepted: 19 August 2011 . Published Online: 19 September 2011

J.-B. Rota, S. Knecht, T. Fleig, D. Ganyushin, T. Saue, F. Neese, and H. Bolvin

ARTICLES YOU MAY BE INTERESTED IN

First-principles calculations of zero-field splitting parameters

The Journal of Chemical Physics 125, 024103 (2006); https://doi.org/10.1063/1.2213976

Efficient and accurate approximations to the molecular spin-orbit coupling operator and their use in molecular $g$-tensor calculations

The Journal of Chemical Physics 122, 034107 (2005); https://doi.org/10.1063/1.1829047

An infinite-order two-component relativistic Hamiltonian by a simple one-step transformation

The Journal of Chemical Physics 126, 064102 (2007); https://doi.org/10.1063/1.2436882

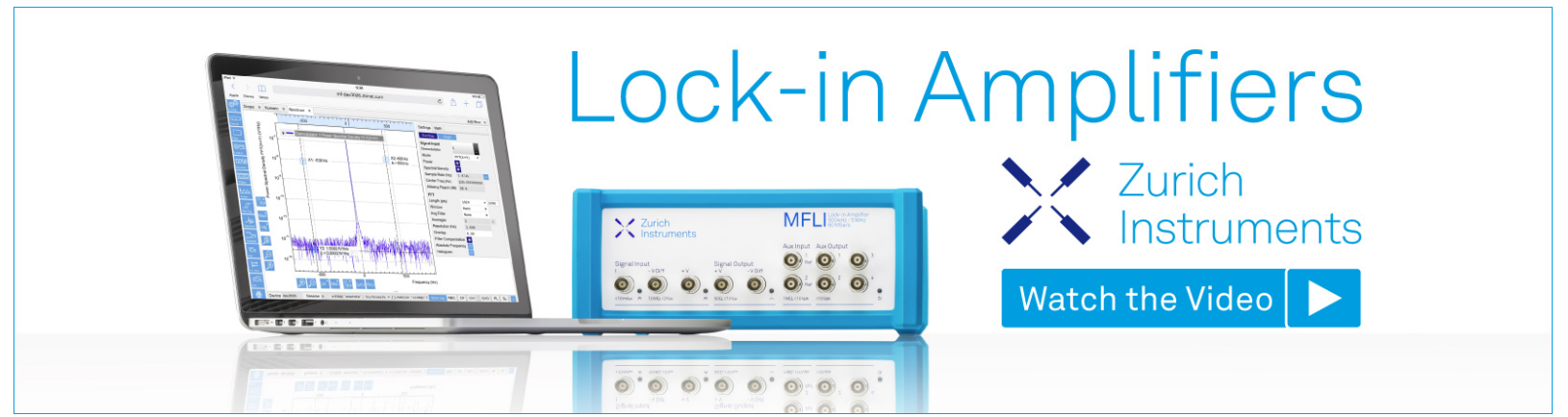

J. Chem. Phys. 135, 114106 (2011); https://doi.org/10.1063/1.3636084

135, 114106

(c) 2011 American Institute of Physics. 


\title{
Zero field splitting of the chalcogen diatomics using relativistic correlated wave-function methods
}

\author{
J.-B. Rota, ${ }^{1}$ S. Knecht, ${ }^{2,3}$ T. Fleig, ${ }^{4}$ D. Ganyushin, ${ }^{5}$ T. Saue ${ }^{2,4}$ F. Neese, ${ }^{5}$ \\ and $\mathrm{H}$. Bolvin $2,4, \mathrm{a})$ \\ ${ }^{1}$ Laboratoire de Chimie, Ecole Normale Supérieure de Lyon, 46 allée d'Italie 69364 Lyon Cédex 07, France \\ ${ }^{2}$ Laboratoire de Chimie Quantique, Institut de Chimie de Strasbourg, 4 rue Blaise \\ Pascal 67000 Strasbourg, France \\ ${ }^{3}$ Department of Physics and Chemistry, Campusvej 55, 5230 Odense M, Denmark \\ ${ }^{4}$ Laboratoire de Chimie et de Physique Quantiques, 118 route de Narbonne, 31062 Toulouse Cédex 04, France \\ ${ }^{5}$ Institut für Physikalische und Theoretische Chemie, Wegelstr. 12, 53115 Bonn, Germany
}

(Received 15 June 2011; accepted 19 August 2011; published online 19 September 2011)

\begin{abstract}
The spectrum arising from the $\left(\pi^{*}\right)^{2}$ configuration of the chalcogen dimers, namely, the $X_{2} 1, a 2$, and $b 0^{+}$states, is calculated using wave-function theory based methods. Two-component (2c) and fourcomponent (4c) multireference configuration interaction (MRCI) and Fock-space coupled cluster (FSCC) methods are used as well as two-step methods spin-orbit complete active space perturbation theory at 2nd order (SO-CASPT2) and spin-orbit difference dedicated configuration interaction (SO-DDCI). The energy of the $X_{2} 1$ state corresponds to the zero-field splitting of the ground state spin triplet. It is described with high accuracy by the 2- and 4-component methods in comparison with experiment, whereas the two-step methods give about $80 \%$ of the experimental values. The $b 0^{+}$ state is well described by 4c-MRCI, SO-CASPT2, and SO-DDCI, but FSCC fails to describe this state and an intermediate Hamiltonian FSCC ansatz is required. The results are readily rationalized by a two-parameter model; $\Delta \epsilon$, the $\pi^{*}$ spinor splitting by spin-orbit coupling and $K$, the exchange integral between the $\pi_{1}^{*}$ and the $\pi_{-1}^{*}$ spinors with, respectively, angular momenta 1 and -1 . This model holds for all systems under study with the exception of $\mathrm{Po}_{2}$. () 2011 American Institute of Physics. [doi:10.1063/1.3636084]
\end{abstract}

\section{INTRODUCTION}

Paramagnetic species with total effective spin $S \geq 1$ may show a zero-field splitting (ZFS) of the ground state: it stems from a splitting of the $2 S+1 M_{S}$ levels even in the absence of an external magnetic field. It determines largely the tunneling barrier and the critical barrier of single molecular magnets, and a rational understanding of the microscopic factors governing the sign and the magnitude of this effect is crucial. ${ }^{1}$ This can be achieved by an accurate description of this effect by quantum mechanical methods. The calculation of ZFS from first principles methods is, however, still a challenging task and calls for benchmark studies of small molecules to establish the methodology. The ZFS has two contributions: (i) the direct dipolar spin-spin coupling that dominates in organic radicals and (ii) the spin-orbit coupling (SOC) contribution that dominates in systems containing heavy atoms. The spin-spin coupling is a first-order term due to the direct dipolar interaction between the spin momenta of two electrons. This contribution was analyzed in the early days of quantum mechanics ${ }^{2,3}$ and has a long history of calculation ${ }^{4-8}$ as well. It is nowadays routinely evaluated from first principles, either using multiconfigurational wave function theory (WFT) (Refs. 9-11) or density functional theory (DFT). ${ }^{12,13}$ It has been shown that this term can exceed $1 \mathrm{~cm}^{-1} .{ }^{14}$ The

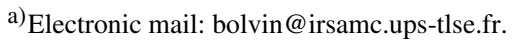

SOC contribution has been analyzed very early by secondorder perturbation theory using crystal-field theory $3,4,15$ and later using ligand-field theory. ${ }^{16}$ The semi-empirical angular orbital model of Bencini is still widely used for transition metal complexes. Recent approaches are based on DFT using the formalism proposed by Pederson and Khanna, ${ }^{17}$ where SOC is taken into account perturbatively. It has been argued underlined by numerical results that the method can be improved by changing some of the prefactors ${ }^{18,19}$ and the formalism has been extended to hybrid functionals solving coupled-perturbed equations. A two-component (2c) noncollinear DFT approach has been proposed by Reviakine et al.: $:^{20}$ in this case, the ZFS is deduced from the difference of energy of two $M_{S}$ components. WFT has been applied by Michl et al. ${ }^{9,21}$ and Ågren et al. ${ }^{10,22}$ on organic radicals using the complete active space self consistent field (CASSCF) method for the calculation of the spin-spin contribution. In two-step methods, the SOC is calculated by a state interaction and the spin-orbit complete active space perturbation theory at 2nd order (SO-CASPT2) method has successfully been applied to the calculation of anisotropic parameters. ${ }^{23-26}$ To the best of our knowledge, $2 \mathrm{c}$ or four-component (4c) relativistic WFT methods have never been applied to the calculation of the ZFS in molecules.

The aim of this article is to assess and compare different WFT methods for the calculation of ZFS from first principles. The chalcogen series has been chosen as benchmark molecules for the following reasons: (i) these molecules are 
small and permit the use of highly accurate methods; (ii) the ZFS is very large at the lower end of the series and comparison to experimental and previous DFT studies is possible. For completeness, we have calculated all the low lying states arising from the $\left(\pi^{*}\right)^{2}$ configuration and compared our results to available experimental data. Two types of methods are considered in this work: (i) one step-methods which are based on the Dirac equation and include SOC variationally from the start, and (ii) two-step methods, where scalar relativistic effects and electron correlation are included in a first step and the SOC treated perturbatively in a second step. In the onestep methods relativistic effects are naturally included, but the inclusion of correlation effects is more expensive due to the reduction of symmetry. We have used $2 \mathrm{c}$ and $4 \mathrm{c}$ general active space configuration interaction (GASCI), Fock-space coupled-cluster (FSCC), and intermediate Hamiltonian Fockspace coupled-cluster (IHFSCC) methods. As two-step methods, spin orbit difference-dedicated configuration interaction (SO-DDCI) and SO-CASPT2 methods have been considered in this work.

The paper is organized as follows: In Sec. II we introduce a two-parameter model which distills the physics of the ZFS in the chalcogen series and is later employed to rationalize our results. In Sec. III a short description of the methods and computational details is given. In Sec. IV A we report calibration studies of two molecules, $\mathrm{Te}_{2}$ and $\mathrm{TeSe}$, studying the effect of the choice of basis set, electron correlation spaces, starting orbitals, and other computational parameters in order to fix these parameters for the rest of the series. In Sec. IV B we report and interpret our results for the complete series in light of our two-parameter model. Final remarks are given in Sec. V.

\section{MODELIZATION OF ZFS}

The chalcogen dimers are open-shell molecules with two electrons in the $\pi_{(g)}^{*}$ orbitals (the subscript $g$ holds for homonuclear molecules and will be omitted in the following). The ground spin-orbit free (SOF) state $X^{3} \Sigma^{-}$is split by ZFS in $\Omega=0^{+}$and $\Omega=1$ states (denoted $X_{1} 0^{+}$and $X_{2} 1$ in spectroscopic literature). The remaining excited states from the $\left(\pi^{*}\right)^{2}$ configuration are $a^{1} \Delta$ and $b^{1} \Sigma^{+}$which become $a 2$ and $b 0^{+}$upon inclusion of SOC.

\section{A. A Hückel model for the construction of molecular spinors}

The aim of this subsection is to build a molecular spinor diagram from the 12 atomic $n p$ valence spinors using a Hückel model. We will, in particular, show that for the systems under study we can neglect second-order SOC which induces mixing of $\sigma$ and $\pi$ orbitals, which we will refer to as the spin-orbit hybridization. We start out in a 2-spinor basis in which the valence atomic spinors $p^{X}\left(j, m_{j}\right)$ localized on the center $X=A, B$ are

$$
p^{X}\left(\frac{1}{2}, \frac{1}{2}\right)=R_{1 / 2}\left(r_{X}\right)\left(\frac{1}{\sqrt{3}} Y_{10}^{X}-\frac{\sqrt{2}}{\sqrt{3}} \bar{Y}_{11}^{X}\right),
$$

$$
\begin{aligned}
p^{X}\left(\frac{1}{2},-\frac{1}{2}\right) & =R_{1 / 2}\left(r_{X}\right)\left(-\frac{1}{\sqrt{3}} \bar{Y}_{10}^{X}+\frac{\sqrt{2}}{\sqrt{3}} Y_{1-1}^{X}\right), \\
p^{X}\left(\frac{3}{2}, \frac{3}{2}\right) & =R_{3 / 2}\left(r_{X}\right) Y_{11}^{X}, \\
p^{X}\left(\frac{3}{2},-\frac{3}{2}\right) & =R_{3 / 2}\left(r_{X}\right) \bar{Y}_{1-1}^{X}, \\
p^{X}\left(\frac{3}{2}, \frac{1}{2}\right) & =R_{3 / 2}\left(r_{X}\right)\left(\frac{\sqrt{2}}{\sqrt{3}} Y_{10}^{X}+\frac{1}{\sqrt{3}} \bar{Y}_{11}^{X}\right), \\
p^{X}\left(\frac{3}{2},-\frac{1}{2}\right) & =R_{3 / 2}\left(r_{X}\right)\left(\frac{\sqrt{2}}{\sqrt{3}} \bar{Y}_{10}^{X}+\frac{1}{\sqrt{3}} Y_{1-1}^{X}\right),
\end{aligned}
$$

where unbarred (barred) spherical harmonics $Y_{l m}$ refer to multiplication with $\alpha(\beta)$ spin.

We first consider the homonuclear case. The $z$ axis is taken as the intermolecular axis. The Hückel Hamiltonian $h$ is defined through its matrix elements:

one-center integrals,

$$
\begin{aligned}
\left\langle p^{A}\right. & \left.\left(\frac{1}{2}, m_{j}\right)|h| p^{A}\left(\frac{1}{2}, m_{j}\right)\right\rangle \\
\quad & =\left\langle p^{B}\left(\frac{1}{2}, m_{j}\right)|h| p^{B}\left(\frac{1}{2}, m_{j}\right)\right\rangle \\
& =\epsilon_{0}-\zeta
\end{aligned}
$$

for $m_{j}= \pm \frac{1}{2}$, where $\zeta$ is the atomic SOC constant.

$$
\begin{aligned}
\left\langle p^{A}\left(\frac{3}{2}, m_{j}\right)|h| p^{A}\left(\frac{3}{2}, m_{j}\right)\right\rangle \\
=\left\langle p^{B}\left(\frac{3}{2}, m_{j}\right)|h| p^{B}\left(\frac{3}{2}, m_{j}\right)\right\rangle \\
=\epsilon_{0}+\frac{1}{2} \zeta
\end{aligned}
$$

for $m_{j}= \pm \frac{3}{2}, \pm \frac{1}{2}$;

two-center integrals,

$$
\begin{aligned}
\left\langle R_{j}\left(r_{A}\right) Y_{10}^{A}|h| R_{j^{\prime}}\left(r_{B}\right) Y_{10}^{B}\right\rangle & =\beta_{j, j^{\prime}}^{\sigma}, \\
\left\langle R_{j}\left(r_{A}\right) Y_{1 \pm 1}^{A}|h| R_{j^{\prime}}\left(r_{B}\right) Y_{1 \pm 1}^{B}\right\rangle & =\beta_{j, j^{\prime}}^{\pi},
\end{aligned}
$$

for $\left(j, j^{\prime}\right) \in\left\{\frac{1}{2}, \frac{3}{2}\right\} . \beta_{j, j^{\prime}}^{\sigma}$ is positive and larger than the absolute value of $\beta_{j, j^{\prime}}^{\pi}$ which is negative.

Note that the two-center integrals are defined with respect to spatial orbitals such that our definition of the Hückel Hamiltonian implies complete neglect of two-center spinorbit interaction. The overlap between orbitals is neglected. The reference energy is taken as $\epsilon_{0}=0$. The Hamiltonian matrix in subspace $m_{j}=\frac{1}{2}$ is 


\begin{tabular}{cccc}
$p^{A}\left(\frac{1}{2}, \frac{1}{2}\right)$ & $p^{A}\left(\frac{3}{2}, \frac{1}{2}\right)$ & $p^{B}\left(\frac{1}{2}, \frac{1}{2}\right)$ & $p^{B}\left(\frac{3}{2}, \frac{1}{2}\right)$ \\
\hline$-\zeta$ & 0 & $\frac{1}{3}\left(\beta_{1 / 2,1 / 2}^{\sigma}+2 \beta_{1 / 2,1 / 2}^{\pi}\right)$ & $\frac{\sqrt{2}}{3}\left(\beta_{1 / 2,3 / 2}^{\sigma}-\beta_{1 / 2,3 / 2}^{\pi}\right)$ \\
0 & $\frac{1}{2} \zeta$ & $\frac{\sqrt{2}}{3}\left(\beta_{1 / 2,3 / 2}^{\sigma}-\beta_{1 / 2,3 / 2}^{\pi}\right)$ & $\frac{1}{3}\left(2 \beta_{3 / 2,3 / 2}^{\sigma}+\beta_{3 / 2,3 / 2}^{\pi}\right)$ \\
$\frac{1}{3}\left(\beta_{1 / 2,1 / 2}^{\sigma}+2 \beta_{1 / 2,1 / 2}^{\pi}\right)$ & $\frac{\sqrt{2}}{3}\left(\beta_{1 / 2,3 / 2}^{\sigma}-\beta_{1 / 2,3 / 2}^{\pi}\right)$ & $-\zeta$ & 0 \\
$\frac{\sqrt{2}}{3}\left(\beta_{1 / 2,3 / 2}^{\sigma}-\beta_{1 / 2,3 / 2}^{\pi}\right)$ & $\frac{1}{3}\left(2 \beta_{3 / 2,3 / 2}^{\sigma}+\beta_{3 / 2,3 / 2}^{\pi}\right)$ & 0 & $\frac{1}{2} \zeta$
\end{tabular}

and in subspace $m_{j}=\frac{3}{2}$ is

$$
\begin{array}{cc}
p^{A}\left(\frac{3}{2}, \frac{3}{2}\right) & p^{B}\left(\frac{3}{2}, \frac{3}{2}\right) \\
\hline \frac{1}{2} \zeta & \beta_{3 / 2,3 / 2}^{\pi} \\
\beta_{3 / 2,3 / 2}^{\pi} & \frac{1}{2} \zeta .
\end{array}
$$

In the following we will assume that the radial parts of $p_{1 / 2}$ and $p_{3 / 2}$ orbitals are identical, that is, we neglect spin-orbit polarization and accordingly set $R_{1 / 2}=R_{3 / 2}=R$, which implies $\beta_{j, j^{\prime}}^{\xi}=\beta^{\xi}$ for $\xi=\sigma, \pi$. This approximation furthermore allows us to transform from the 2-spinor atomic basis to a basis of pure spin molecular orbitals:

$$
\begin{aligned}
& \pi_{3 / 2}=\frac{1}{\sqrt{2}}\left(R\left(r_{A}\right) Y_{11}^{A}+R\left(r_{B}\right) Y_{11}^{B}\right), \\
& \pi_{3 / 2}^{*}=\frac{1}{\sqrt{2}}\left(R\left(r_{A}\right) Y_{11}^{A}-R\left(r_{B}\right) Y_{11}^{B}\right), \\
& \pi_{1 / 2}=\frac{1}{\sqrt{2}}\left(R\left(r_{A}\right) \bar{Y}_{11}^{A}+R\left(r_{B}\right) \bar{Y}_{11}^{B}\right), \\
& \pi_{1 / 2}^{*}=\frac{1}{\sqrt{2}}\left(R\left(r_{A}\right) \bar{Y}_{11}^{A}-R\left(r_{B}\right) \bar{Y}_{11}^{B}\right), \\
& \sigma_{1 / 2}=\frac{1}{\sqrt{2}}\left(R\left(r_{A}\right) Y_{10}^{A}-R\left(r_{B}\right) Y_{10}^{B}\right), \\
& \sigma_{1 / 2}^{*}=\frac{1}{\sqrt{2}}\left(R\left(r_{A}\right) Y_{10}^{A}+R\left(r_{B}\right) Y_{10}^{B}\right) .
\end{aligned}
$$

Matrices (5) and (6) become after corresponding rotation

$$
\begin{array}{cccc}
\pi_{1 / 2}^{*} & \sigma_{1 / 2} & \pi_{1 / 2} & \sigma_{1 / 2}^{*} \\
\hline-\beta^{\pi}-\frac{1}{2} \zeta & \frac{1}{\sqrt{2}} \zeta & 0 & 0 \\
\frac{1}{\sqrt{2}} \zeta & -\beta^{\sigma} & 0 & 0 \\
0 & 0 & \beta^{\pi}-\frac{1}{2} \zeta & \frac{1}{\sqrt{2}} \zeta \\
0 & 0 & \frac{1}{\sqrt{2}} \zeta & \beta^{\sigma} \\
& & \\
\pi_{3 / 2}^{*} & \pi_{3 / 2} \\
\hline-\beta^{\pi}+\frac{1}{2} \zeta & 0 \\
0 & \beta^{\pi}+\frac{1}{2} \zeta
\end{array}
$$

Matrices (8) and (9) are both diagonal when $\zeta=0$. The effect of SOC is at 1 st order to split the $\pi_{1 / 2}^{(*)}-\pi_{3 / 2}^{(*)}$ orbitals by $\zeta$, the $\pi_{1 / 2}^{(*)}$ being the lowest. At 2 nd order, it mixes bonding $\sigma_{1 / 2}$ $\left(\pi_{1 / 2}\right)$ with anti-bonding $\pi_{1 / 2}^{*}\left(\sigma_{1 / 2}^{*}\right)$ orbitals, which we refer to as the spin-orbit hybridization. There is neither $\pi_{1 / 2} / \sigma_{1 / 2}$ nor $\pi_{1 / 2}^{*} / \sigma_{1 / 2}^{*}$ mixing because these orbitals have opposite inversion symmetry. The $\sigma-\pi$ mixing due to SOC can be evaluated pertubatively as $\frac{\zeta^{2}}{2\left(\beta^{\sigma}+\left|\beta^{\pi}\right|\right)^{2}}$ (the 1 st order SOC is neglected in the denominator). $\beta^{\sigma}$ and $\beta^{\pi}$ can be estimated from
CASSCF calculations. We have calculated them as half the difference of the canonical energies of the antibonding and the corresponding bonding orbitals using complete active space $(\mathrm{CAS})(8,6)$ and all-root state-averaged $\operatorname{CAS}(8,6)$ calculations. The constants $\zeta$ are evaluated in the Appendix. In Table I we report our calculated parameters $\zeta, \beta^{\sigma}$, and $\beta^{\pi}$ and the $\sigma-\pi$ hybridization predicted from our Hückel model. In practice, we find that the SO hybridization is negligible for all systems except $\mathrm{Po}_{2}$ where the $\sigma$ orbital contributes $7.8 \%$ to the $\pi_{1 / 2}$ orbital.

For heteronuclear molecules, there is no inversion center. Let $2 \delta \epsilon$ be the energy gap between SOF $n p$ orbitals of $A$ and $B$ and $\delta \zeta$ be the difference between the two atomic SOC constants. Equations (2) and (3) become

$$
\begin{aligned}
\left\langle p^{A}\left(\frac{1}{2}, m_{j}\right)|h| p^{A}\left(\frac{1}{2}, m_{j}\right)\right\rangle & =\epsilon_{0}+\delta \epsilon-(\zeta+\delta \zeta), \\
\left\langle p^{B}\left(\frac{1}{2}, m_{j}\right)|h| p^{B}\left(\frac{1}{2}, m_{j}\right)\right\rangle & =\epsilon_{0}-\delta \epsilon-(\zeta-\delta \zeta), \\
\left\langle p^{A}\left(\frac{3}{2}, m_{j}\right)|h| p^{B}\left(\frac{3}{2}, m_{j}\right)\right\rangle & =\epsilon_{0}+\delta \epsilon+\frac{1}{2}(\zeta+\delta \zeta), \\
\left\langle p^{B}\left(\frac{3}{2}, m_{j}\right)|h| p^{B}\left(\frac{3}{2}, m_{j}\right)\right\rangle & =\epsilon_{0}-\delta \epsilon+\frac{1}{2}(\zeta-\delta \zeta) .
\end{aligned}
$$

The SOF eigenvectors of matrices (5) and (6) are

$$
\begin{aligned}
& \pi_{3 / 2}=\sin \frac{\theta}{2} R\left(r_{A}\right) Y_{11}^{A}+\cos \frac{\theta}{2} R\left(r_{B}\right) Y_{11}^{B}, \\
& \pi_{3 / 2}^{*}=\cos \frac{\theta}{2} R\left(r_{A}\right) Y_{11}^{A}-\sin \frac{\theta}{2} R\left(r_{B}\right) Y_{11}^{B}, \\
& \pi_{1 / 2}=\sin \frac{\theta}{2} R\left(r_{A}\right) \bar{Y}_{11}^{A}+\cos \frac{\theta}{2} R\left(r_{B}\right) \bar{Y}_{11}^{B},
\end{aligned}
$$

TABLE I. Hückel parameters $\beta^{\sigma}$ and $\beta^{\pi}$ deduced from CASSCF calculations, SOC constant $\zeta . \sigma-\pi$ mixing and energy shift $\delta \epsilon$ due to $\sigma / \pi$ hybridization. All energies are in $\mathrm{cm}^{-1}$.

\begin{tabular}{lccrcccr}
\hline \hline & & & & & \multicolumn{3}{c}{ Hybridization } \\
& $\beta^{\sigma}$ & \multicolumn{1}{c}{$\beta^{\pi}$} & \multicolumn{1}{c}{$\zeta$} & $\frac{\left|\beta^{\pi}\right|}{\beta^{\sigma}}$ & $\frac{\zeta}{\beta^{\sigma}}$ & $\sigma-\pi(\%)$ & $\delta \epsilon$ \\
\hline $\mathrm{S}_{2}$ & 44500 & -19200 & 250 & 0.43 & 0.009 & 0.002 & 1 \\
$\mathrm{Se}_{2}$ & 36500 & -14500 & 1200 & 0.40 & 0.06 & 0.05 & 32 \\
$\mathrm{Te}_{2}$ & 28700 & -10600 & 3138 & 0.37 & 0.09 & 0.2 & 87 \\
$\mathrm{Po}_{2}$ & 24900 & -8800 & 12369 & 0.35 & 0.49 & 7.8 & 2545 \\
\hline \hline
\end{tabular}




$$
\begin{aligned}
& \pi_{1 / 2}^{*}=\cos \frac{\theta}{2} R\left(r_{A}\right) \bar{Y}_{11}^{A}-\sin \frac{\theta}{2} R\left(r_{B}\right) \bar{Y}_{11}^{B}, \\
& \sigma_{1 / 2}=\sin \frac{\phi}{2} R\left(r_{A}\right) Y_{10}^{A}-\cos \frac{\phi}{2} R\left(r_{B}\right) Y_{10}^{B}, \\
& \sigma_{1 / 2}^{*}=\cos \frac{\phi}{2} R\left(r_{A}\right) Y_{10}^{A}+\sin \frac{\phi}{2} R\left(r_{B}\right) Y_{10}^{B}
\end{aligned}
$$

with

$$
\tan \phi=\frac{-\beta^{\pi}}{\delta \epsilon}
$$

$$
\tan \theta=\frac{\beta^{\sigma}}{\delta \epsilon}
$$

\begin{tabular}{|c|c|c|c|}
\hline$\pi_{1 / 2}^{*}$ & $\sigma_{1 / 2}$ & $\pi_{1 / 2}$ & $\sigma_{1 / 2}^{*}$ \\
\hline$-\sin \theta \beta^{\pi}+\cos \theta \delta \epsilon-\frac{1}{2} \zeta-\frac{1}{2} \cos \theta \delta \zeta$ & $\frac{1}{\sqrt{2}} \sin \frac{1}{2}(\phi+\theta)(\zeta+\delta \zeta)$ & $-\frac{1}{2} \sin \theta \delta \zeta$ & $\frac{1}{\sqrt{2}} \cos \frac{1}{2}(\phi+\theta)(\zeta+\delta \zeta)$ \\
\hline$\frac{1}{\sqrt{2}} \sin \frac{1}{2}(\phi+\theta)(\zeta+\delta \zeta)$ & $-\sin \phi \beta^{\sigma}-\cos \phi \delta \epsilon$ & $\frac{1}{\sqrt{2}} \cos \frac{1}{2}(\phi+\theta)(-\zeta+\delta \zeta)$ & 0 \\
\hline$-\frac{1}{2} \sin \theta \lambda$ & $\frac{1}{\sqrt{2}} \cos \frac{1}{2}(\phi+\theta)(-\zeta+\delta \zeta)$ & $\sin \theta \beta^{\pi}-\cos \theta \delta \epsilon-\frac{1}{2} \zeta+\frac{1}{2} \cos \theta \delta \zeta$ & $\frac{1}{\sqrt{2}} \sin \frac{1}{2}(\phi+\theta)(\zeta-\delta \zeta)$ \\
\hline$\frac{1}{\sqrt{2}} \cos \frac{1}{2}(\phi+\theta)(\zeta+\delta \zeta)$ & 0 & $\frac{1}{\sqrt{2}} \sin \frac{1}{2}(\phi+\theta)(\zeta-\delta \zeta)$ & $\sin \phi \beta^{\sigma}+\cos \phi \delta \epsilon$ \\
\hline \multicolumn{2}{|c|}{$\pi_{3 / 2}^{*}$} & \multicolumn{2}{|l|}{$\pi_{3 / 2}$} \\
\hline $\begin{array}{r}-\sin \theta \beta^{\pi}+\cos \theta \delta \epsilon \\
\frac{1}{2} \sin \theta\end{array}$ & $\begin{array}{l}+\frac{1}{2} \zeta+\frac{1}{2} \cos \theta \delta \zeta \\
\delta \zeta\end{array}$ & $\begin{array}{r}\frac{1}{2} \sin \theta \delta \zeta \\
\sin \theta \beta^{\pi}-\cos \theta \delta \epsilon+\frac{1}{2}\end{array}$ & $-\frac{1}{2} \cos \theta \delta \zeta$ \\
\hline
\end{tabular}

The solutions of the homonuclear case are found again for $\phi=\theta=\frac{\pi}{2}$. On the basis of Eqs. (11), matrices (5) and (6) become
In heteronuclear molecules, the inversion center is removed and consequently the $g, u$ symmetry. There is hybridization by $\sigma^{*}-\pi^{*}$ and $\sigma-\pi$ couplings and those coupling terms become predominant in the case of large difference of electronegativity $2 \delta \varepsilon$ between the two atoms. At the limit, $\theta=\phi=0$, one retrieves the atomic solutions of Eq. (10). In the case of our most asymmetric molecule, TeO, $\delta \epsilon=18585 \mathrm{~cm}^{-1}$ as evaluated from ionization energies. The $\beta$ are taken as mean values of the two species, one finds $\phi=31^{\circ}, \theta=19^{\circ}$, and matrices (13) and (14) are (in $\mathrm{cm}^{-1}$ )

\begin{tabular}{cccc}
$\pi_{1 / 2}^{*}$ & $\sigma_{1 / 2}$ & $\pi_{1 / 2}$ & $\sigma_{1 / 2}^{*}$ \\
\hline 22410 & 1145 & -450 & 1755 \\
1145 & -41050 & 240 & 0 \\
-450 & 240 & -24105 & 715 \\
1755 & 0 & 715 & 41050 \\
& & & \\
& $\pi_{3 / 2}^{*}$ & $\pi_{3 / 2}$ & \\
& 24230 & 450 \\
& 450 & -23535
\end{tabular}.

These matrices are still dominated by the diagonal energies determined by the internuclear $\beta$ parameters. The $\sigma^{*}-\pi^{*}$ mixing of the wave function is $0.9 \%$.

The model can be improved by incorporating SO polarization that will increase the $\sigma-\pi$ hybridization. According to the present model, SO hybridization is small, and to a good approximation, the " $\pi^{*}$ " spinors can be considered to be pure $\pi$ spin orbitals. According to Eqs. (11), one can write

$$
\begin{aligned}
\pi_{3 / 2}^{*}=\phi_{1} & =\cos \frac{\theta}{2} R\left(r_{A}\right) Y_{11}^{A}-\sin \frac{\theta}{2} R\left(r_{B}\right) Y_{11}^{B}, \\
\pi_{-3 / 2}^{*}=\bar{\phi}_{-1} & =\cos \frac{\theta}{2} R\left(r_{A}\right) \bar{Y}_{1-1}^{A}-\sin \frac{\theta}{2} R\left(r_{B}\right) \bar{Y}_{1-1}^{B}, \\
\pi_{1 / 2}^{*}=\bar{\phi}_{1} & =\cos \frac{\theta}{2} R\left(r_{A}\right) \bar{Y}_{11}^{A}-\sin \frac{\theta}{2} R\left(r_{B}\right) \bar{Y}_{11}^{B}, \\
\pi_{-1 / 2}^{*}=\phi_{-1} & =\cos \frac{\theta}{2} R\left(r_{A}\right) Y_{1-1}^{A}-\sin \frac{\theta}{2} R\left(r_{B}\right) Y_{1-1}^{B},
\end{aligned}
$$

where $\phi_{ \pm 1}\left(\bar{\phi}_{ \pm 1}\right)$ is a pure spin spinor with spin $\alpha(\beta)$ and spatial part $\tilde{\phi}_{ \pm 1}$ with angular momentum along the molecular axis $M_{L}= \pm 1$.

\section{B. A two-parameter model of ZFS}

As shown in Subsection II A, for all systems except $\mathrm{Po}_{2}$, the bonding $\sigma$-contributions to the four $\pi^{*}$ spinors can be neglected and the $\pi^{*}$ spinors are thus pure spin spinors. Using these spinors, within the $\left(\pi^{*}\right)^{2}$ configuration, the following determinants $\left|M_{J}\right\rangle$ can be built:

symmetry $\Omega=0$,

$$
\begin{aligned}
& |0\rangle=\left|\pi_{1 / 2}^{*} \pi_{-1 / 2}^{*}\right|=\left|\bar{\phi}_{1} \phi_{-1}\right|, \\
& \left|0^{*}\right\rangle=\left|\pi_{3 / 2}^{*} \pi_{-3 / 2}^{*}\right|=\left|\phi_{1} \bar{\phi}_{-1}\right|
\end{aligned}
$$

symmetry $\Omega=1$,

$$
\begin{aligned}
& |+1\rangle=\left|\pi_{3 / 2}^{*} \pi_{-1 / 2}^{*}\right|=\left|\phi_{1} \phi_{-1}\right|, \\
& |-1\rangle=\left|\pi_{-3 / 2}^{*} \pi_{1 / 2}^{*}\right|=\left|\bar{\phi}_{-1} \bar{\phi}_{1}\right|,
\end{aligned}
$$

symmetry $\Omega=2$,

$$
\begin{gathered}
|+2\rangle=\left|\pi_{3 / 2}^{*} \pi_{1 / 2}^{*}\right|=\left|\phi_{1} \bar{\phi}_{1}\right|, \\
|-2\rangle=\left|\pi_{-3 / 2}^{*} \pi_{-1 / 2}^{*}\right|=\left|\bar{\phi}_{-1} \phi_{-1}\right| .
\end{gathered}
$$

$\Delta \varepsilon=\varepsilon_{3 / 2}-\varepsilon_{1 / 2}$ is the energy gap between the $\pi_{3 / 2}^{*}$ and $\pi_{1 / 2}^{*}$ spinors. Its value is about the same order of magnitude as $\zeta$, the SOC constant in the case of homonuclear molecules (see Appendix). $J=\left(\tilde{\phi}_{1} \tilde{\phi}_{1} \mid \tilde{\phi}_{-1} \tilde{\phi}_{-1}\right)$ and $K$ 
$=\left(\tilde{\phi}_{1} \tilde{\phi}_{-1} \mid \tilde{\phi}_{1} \tilde{\phi}_{-1}\right)$ are the Coulomb and exchange twoelectron integrals (Mulliken notations have been used). The energy of the $b 2$ and $a 1$ states is $\varepsilon_{1 / 2}+\varepsilon_{3 / 2}+J$ and $\varepsilon_{1 / 2}$ $+\varepsilon_{3 / 2}+J-K$, respectively, while the energies of the states corresponding to the $\Omega=0$ states are obtained as the eigenvalues of the following matrix:

$$
\begin{array}{cc}
|0\rangle & \left|0^{*}\right\rangle \\
\hline 2 \varepsilon_{1 / 2}+J & -K \\
-K & 2 \varepsilon_{3 / 2}+J
\end{array} .
$$

Finally, the energies of the four states become, after shifting the zero point of the energy to $\varepsilon_{3 / 2}+\varepsilon_{1 / 2}+J$,

$$
\begin{gathered}
E_{b 0^{+}}=\sqrt{\Delta \varepsilon^{2}+K^{2}}, \\
E_{a 2}=0, \\
E_{X_{2} 1}=-K, \\
E_{X_{1} 0^{+}}=-\sqrt{\Delta \varepsilon^{2}+K^{2}} .
\end{gathered}
$$

The ZFS is then calculated as the difference between the energies of the two lowest states $X_{1} 0^{+}$and $X_{2} 1$ :

$$
D=-K+\sqrt{\Delta \varepsilon^{2}+K^{2}} .
$$

Within the two-step approach, one considers the SOF functions, $\left|X_{1}{ }^{3} \Sigma^{-} ; M_{S}=0\right\rangle=\frac{1}{\sqrt{2}}\left(|0\rangle-\left|0^{*}\right\rangle\right)$ and $\left|b^{1} \Sigma^{+}\right\rangle$ $=\frac{1}{\sqrt{2}}\left(|0\rangle+\left|0^{*}\right\rangle\right)$ and on this basis, the Hamiltonian matrix becomes (keeping the same energy origin)

$$
\frac{\left|X^{3} \Sigma^{-} ; M_{S}=0\right\rangle\left|b^{1} \Sigma^{+}\right\rangle}{-K} \quad \begin{gathered}
\Delta \varepsilon \\
\Delta \varepsilon
\end{gathered}
$$

Without SOC, $\Delta \varepsilon=0$, and the $\left|{ }^{3} \Sigma^{-} ; M_{S}=0\right\rangle$ and $\left.\left.\right|^{3} \Sigma_{(g)}^{-} ; M_{S}= \pm 1\right\rangle$ states are degenerate, as expected.

To conclude this section, the ZFS within the $\left(\pi^{*}\right)^{2}$ configuration is governed by the competitive interplay of the SOC splitting $\Delta \varepsilon$ that favours the closed shell $\left(\pi_{1 / 2}^{*}\right)^{2}$ state and the exchange energy $K$ between the $\pi_{1}^{*}$ and $\pi_{-1}^{*}$ with current circulating in opposite sense which tends to give the ground state as a two-determinant state degenerate with the $1_{(g)}$ state. Both spin-orbit polarization and spin-orbit hybridization have been neglected in this model. With spin-orbit polarization, $\pi_{1 / 2}^{*}$ and $\pi_{3 / 2}^{*}$ do not have the same radial dependence and it adds some Coulomb contribution to the ZFS expression. Spin-orbit hybridization mixes a $\sigma$ contribution into the above " $\pi_{1 / 2}^{*}$ " spinor such that it is no longer a pure spin $\pi^{*}$ spinor.

\section{COMPUTATIONAL DETAILS}

\section{A. One-step SOC methods}

One-step SOC calculations were carried out with the DIRAC10 (Ref. 27) program. Four-component calculations are based on the Dirac-Coulomb Hamiltonian which comprises spin-orbit interaction induced by the relative motion of electrons with respect to the nuclei as well as spin-same orbit (SSO) interaction between electrons. Calculations were also carried out with the molecular mean-field (mmf) DiracCoulomb-Gaunt (DCG) Hamiltonian, ${ }^{28}$ where the Gaunt term adds spin-other orbit (SOO) interaction (as well as spin-spin interaction). As a further approximation we eliminated all two-electron integrals containing small components from the 4-index transformation, thus providing a significant speedup. In the notation used in Ref. 28, this constitutes the mmf${ }^{4} \mathrm{DCG}^{* *}$ scheme. The latter approximation was carefully tested for the molecules studied in this paper, and was found to give negligible errors.

Two-component calculations were based on the one-step eXact 2-component (X2C) relativistic Hamiltonian of Iliaš and Saue. ${ }^{29}$ Two-electron SSO and SOO contributions were included via atomic mean-field integrals (AMFI). ${ }^{30,31}$

\section{FSCC and IHFSCC}

The FSCC (Ref. 32) method is a multireference coupled cluster method and is fully size-extensive for both ground and excited states, including electron correlation to infinite order. In order to use this method one needs to start from a closed-shell reference which in our case can be either the di-anionic or di-cationic system. The FSCC schemes for the dianion calculations can be written as: $X Y^{2-}(0 h, 0 p)$ $\rightarrow X Y^{-}(1 h, 0 p) \rightarrow X Y(2 h, 0 p)$. For the heaviest dimers $\left(\mathrm{Po}_{2}\right.$ and $\left.\mathrm{Te}_{2}\right)$, one may also start from the neutral system because in its ground state these molecules are closed-shell systems. The FSCC schemes for the neutral calculations can, for example, similarly be written as: $X Y(0 h, 0 p)$ $\rightarrow\left\{X Y^{+}(1 h, 0 p), X Y^{-}(0 h, 1 p)\right\} \rightarrow X Y^{*}(1 h, 1 p)$. From this it follows that only monoexcitations compared to the $\left|\pi_{1 / 2} \pi_{-1 / 2}\right|$ determinant are considered and that the $X_{1} 0^{+}$ state is described by a single determinant, whereas the $b 0^{+}$ state cannot be described at all. The total correlation space comprises in either scheme the $n p_{\sigma}$ and $n p_{\pi}$ spinors and inner valence $n s$ and $(n-1) d$ shells in combination with the $n p_{\pi^{*}}$ spinors in the active model space.

Seizing the suggestions of one of the referees, further calculations have been performed within the IHFSCC ansatz. ${ }^{32-34}$ In these calculations, carried out in the framework of the above described dianion approach, two total active spaces $P=P_{m}+P_{I}$ have been considered, where $P_{m}$ and $P_{I}$ denote the model and intermediate spaces, respectively. In the scheme denoted [a], $P_{m}=\left[n p_{\pi^{*}}\right]$ and $P_{I}=\left[n p_{\sigma}, n p_{\pi}\right]$, while the electrons of the inner valence $n s$ and $(n-1) d$ shells are correlated. In the scheme denoted [b], $P_{m}=\left[n p_{\sigma}, n p_{\pi}, n p_{\pi^{*}}\right]$ and $P_{I}=[n s,(n-1) d]$. In both schemes, 32 electrons are correlated but the larger active space used in scheme [b] permits a better relaxation of the wave function with correlation. As suggested by our referee, we have considered two sets of spinors: either the canonical spinors of the $X Y^{2-}$ dianion or the spinors obtained by an average-of-configuration HF calculation obtained with 2 electrons in 4 spinors $\left(\pi_{1 / 2}^{*}, \pi_{3 / 2}^{*}\right)$.

Triple zeta (TZ), and quadruple zeta (QZ) basis sets of Dyall ${ }^{35}$ for the heavier and Dunning-type basis sets for the lighter elements, respectively, augmented with diffuse 
functions were employed in uncontracted form in all (IH)FSCC calculations, which typically necessitates the use of a cutoff in the virtual space. We set the threshold to about 46 hartree, such that all recommended core-valence and valence-correlation functions as well as the diffuse functions were included in the virtual correlation space. Calculations with the QZ basis sets were necessarily performed at the 4c level since the AMFI code interfaced to DIRAC10 cannot handle $h$ functions.

\section{GASCI}

GASCI calculations have been carried out with the relativistic double group configuration interaction (CI) program LUCIAREL (Ref. 36) which recently has been extended ${ }^{37}$ for parallel computer applications implemented in DIRAC10. The molecular spinors for the CI calculations have been obtained by an average-of-configurations Hartree-Fock calculation, where the open shells are defined as the two partially occupied Kramers pairs of $\pi^{*}$ character as for IHFSCC calculations. Using the concept of general active space, ${ }^{38}$ two correlation schemes were constructed. In the first (second) scheme denoted v-GASCI (cv-GASCI), the 12 (32) electrons of the $n s$ and $n p$ (and $(n-1) d$ ) shells are correlated. The orbital space is subdivided into a CAS $(6,4)$ comprising 6 electrons in the $n p_{\pi}$ and $n p_{\pi^{*}}$ spinors and a second orbital space comprising the $n p_{\sigma}, n s$ (and $(n-1) d$ ) spinors, the latter being restricted to 0,1 , and 2 holes. From the total reference space, single and double excitations are allowed. The TZ and QZ basis sets of Dyall ${ }^{35}$ were employed in uncontracted form. In the case of $\mathrm{Te}_{2}$, valence correlating functions were added to the basis set, and the cutoff for virtual spinors was set to 10 a.u. For all the other molecules treated with GASCI, valence- and core-correlating functions were added, and the cutoff of the virtual was set to 20 a.u.

\section{B. Two-step SOC methods}

\section{1. $S O-D D C I$}

SO-DDCI calculations were performed with the program ORCA 2.7.0. ${ }^{39}$ The ORCA implementation of DDCI is based on spin-adapted configuration state functions rather than individual determinants and is described in Ref. 40. No symmetry is used in the calculations. SOF calculations are performed at the CAS + DDCIn level. ${ }^{41}$ Scalar relativistic effects are included using either the secondorder Douglas-Kroll-Hess (DKH) Hamiltonian ${ }^{42}$ or the zeroth order regular approximation (ZORA) Hamiltonian. ${ }^{43}$ The spin orbit mean field operator (SOMF) was used; it differs from the AMFI by avoiding the one-center and frozen atomic density approximations as described in Ref. 18. Basis sets of TZP quality are used; ${ }^{44}$ calculations with basis sets of QZ quality were not possible for technical reasons.

Orbitals were optimized by an average CASSCF calculation on 20 triplet and 20 singlet states. $\operatorname{A~CAS}(6,4)$ is used as in the GASCI calculations specified in Subsection III A. All occupied and virtual orbitals were taken into account and, consequently, all the electrons. Different levels of correlation are taken into account; the CAS + DDCIn space is formed by the determinants such as $n_{h}$ being the total number of holes in the occupied and $n_{p}$ being the number of particles in the virtual is $n_{h}+n_{p} \leq n$ with $n_{h} \leq 2$ and $n_{p} \leq 2$. When $n=4$, it is a CAS + SD space. Furthermore, determinants are preselected by a perturbative criterion with a coefficient threshold $T_{\text {pre }}$ of $10^{-10}$ hartree. Test calculations have revealed that this threshold provides converged results. The SOC is calculated as the state interaction between the 40 SOF states.

\section{SO-CASPT2}

SO-CASPT2 calculations were performed with the MOLCAS7 package. ${ }^{45}$ The atomic natural orbital (ANO)-RCC (Refs. 46 and 47) basis functions were used with the standard TZP and QZP contractions. Scalar relativistic effects were included using the second-order DKH transformation. Wave functions have been determined by state-averaged CASSCF calculations. ${ }^{48}$ Three active spaces are considered; CAS $(2,2)$ with 2 electrons in the $\pi^{*}$ orbitals, $\operatorname{CAS}(6,4)$ with 6 electrons in the $\pi$ and $\pi^{*}$ orbitals, and $\operatorname{CAS}(8,6)$ with 8 electrons in the orbitals issued from all the atomic $n p$ orbitals. All the SOF roots up till $70000 \mathrm{~cm}^{-1}$ have been taken into account. Dynamical correlation is included using the multi state-CASPT2 method ${ }^{49}$ using the default values for the IPEA shift and the frozen orbitals. SOC is calculated as a state interaction between the SOF states using SO-RASSI (Ref. 50) and AMFI. ${ }^{30,31}$ For SO-CASPT2, the state interaction is calculated with the wavefunctions obtained by the MS-CASPT2 procedure.

\section{RESULTS AND DISCUSSIONS}

Our series of diatomic molecules, with the exception of $\mathrm{Po}_{2}$, is well characterized by spectroscopy. Relevant experimental data is listed in Table VI. It should be noted that many values of the energies of the $X_{2} 1$ state differ from the experimental values given in Refs. 18,20, which employed older data taken from Ref. 51. The ZFS varies by three orders of magnitude between $\mathrm{O}_{2}$ and $\mathrm{Te}_{2}$ and different techniques have been used in order to measure it. Rotational spectroscopy brings insights into the components of the ground state $X^{3} \Sigma^{-}$. This splitting is sufficiently small in $\mathrm{O}_{2}$ and $\mathrm{Se}_{2}$ to be directly measured by electron paramagnetic resonance. Rotational bands are split due to spin-rotation coupling and this energy splitting is related to the ZFS. The coupling scheme according to Hund's classification varies within the series from case $\mathrm{b}$ to case $\mathrm{a}$ and $\mathrm{c}$ for the heaviest elements ${ }^{52}$ and the ZFS is deduced from the $\Omega$ doubling of the rotational bands. The $b^{1} \Sigma^{+}$to the $X^{3} \Sigma^{-}$transitions can be observed by chemiluminescence. These bands are in the near infrared: while these transitions are electric dipole allowed for heteronuclear molecules, only the $b 0_{b}^{+} \rightarrow X_{2} 1_{g}$ magnetic dipole transition is allowed for the homonuclear species. ${ }^{53-59}$ This method has permitted the detection of the $a^{1} \Delta \rightarrow X^{3} \Sigma^{-}$ transition in $\mathrm{SO}$ and $\mathrm{S}_{2}$. Finally, in the emission spectra using high-resolution Fourier transform laser fluorescence spectroscopy, the $B 0_{u}^{+} \rightarrow b^{1} \Sigma_{g}^{+}, B 0_{u}^{+} \rightarrow X 1_{g}$ and $B 0_{u}^{+}$ 
TABLE II. First excited states energies (in $\mathrm{cm}^{-1}$ ) of $\mathrm{Te}_{2}$ calculated with $2 \mathrm{c}$ and $4 \mathrm{c}$ methods. For $\alpha^{2}$, see Sec. IV A 1.

\begin{tabular}{|c|c|c|c|c|c|c|c|c|}
\hline Method & Gaunt & $\#$ corr. $\mathrm{e}^{-}$ & Options & Basis set & $X_{2} 1_{g}$ & $a 2_{g}$ & $b 0_{g}^{+}$ & $\alpha^{2}$ \\
\hline Expt. & & & & & 1975 & & 9600 & \\
\hline \multirow[t]{2}{*}{ 2c-GASCI } & yes & $12 \mathrm{e}$ & & $\mathrm{TZ}$ & 1836 & 5050 & 9664 & \\
\hline & & $32 \mathrm{e}$ & & $\mathrm{TZ}$ & 1929 & 5037 & 9717 & \\
\hline \multirow[t]{2}{*}{ 4c-GASCI } & no & $32 \mathrm{e}$ & & $\mathrm{TZ}$ & 1964 & 5069 & 9783 & \\
\hline & & $32 \mathrm{e}$ & & QZ & 2018 & 5005 & 9743 & 0.889 \\
\hline \multirow[t]{5}{*}{ 2c-FSCC } & yes & $32 \mathrm{e}$ & dication & $\mathrm{TZ}$ & 1537 & 4811 & 10570 & 0.843 \\
\hline & yes & $32 \mathrm{e}$ & neutral & $\mathrm{TZ}$ & 1898 & 6549 & $\ldots$ & 1 \\
\hline & yes & $12 \mathrm{e}$ & dianion & $\mathrm{TZ}$ & 1685 & 5663 & 12310 & 0.836 \\
\hline & yes & $32 \mathrm{e}$ & dianion & $\mathrm{TZ}$ & 1840 & 5754 & 12351 & 0.848 \\
\hline & no & $32 \mathrm{e}$ & dianion & $\mathrm{TZ}$ & 1874 & 5785 & 12417 & \\
\hline \multirow[t]{2}{*}{ 4c-FSCC } & yes & $32 \mathrm{e}$ & dianion & $\mathrm{TZ}$ & 1839 & 5752 & 12348 & 0.848 \\
\hline & yes & $32 \mathrm{e}$ & dianion & QZ & 1959 & 5769 & 12507 & 0.854 \\
\hline \multirow[t]{3}{*}{ 4c-IHFSCC } & yes & $32 \mathrm{e}$ & [a]/dianion spinors & QZ & 2105 & 5563 & 11649 & 0.869 \\
\hline & yes & $32 \mathrm{e}$ & {$[a] /$ neutral spinors } & QZ & 2128 & 5046 & 10289 & 0.894 \\
\hline & yes & $32 \mathrm{e}$ & {$[\mathrm{b}] /$ neutral spinors } & QZ & 2178 & 5029 & 9724 & 0.898 \\
\hline
\end{tabular}

$\rightarrow X 0_{g}^{+}$transitions have been observed for $\mathrm{Se}_{2}, \mathrm{TeSe}$, and $\mathrm{Te}_{2} .{ }^{60-62}$ The $a^{1} \Delta$ state has not been observed for the heaviest elements of the series.

Experimental bond lengths taken from Ref. 51 have been employed throughout this work, except for $\mathrm{Po}_{2}$ for which no experimental data are available. For this molecule, we performed a geometry optimization at the $2 \mathrm{c}$-coupled-cluster single double triple level using the augmented TZ basis including both SSO and SOO contributions. We obtained an equilibrium bond distance $R_{e}$ of $2.795 \AA$ and a harmonic frequency $\omega_{e}$ of $155.6 \mathrm{~cm}^{-1}$. In Sec. IV A we report calibration studies of the homonuclear $\mathrm{Te}_{2}$ and the heteronuclear TeSe molecules playing extensively with the computational parameters. Then, in Sec. IV B we present and discuss our results for the whole series calculated with the chosen set of parameters.

\section{A. Calibration studies of $\mathrm{Te}_{2}$ and $\mathrm{TeSe}$}

\section{One-step SOC methods}

Results are summarized in Tables II and III. $\alpha^{2}$ is the renormalized weight of the $\left|\pi_{1 / 2}^{*} \pi_{-1 / 2}^{*}\right|$ determinant in the $X_{1} 0_{g}$ state. The calculations performed at the best level of each theory compare very well to the experimental values. With both GASCI and FSCC methods, the comparison be-

TABLE III. First excited states energies (in $\mathrm{cm}^{-1}$ ) of TeSe calculated with $2 \mathrm{c}$ and $4 \mathrm{c}$ methods. For $\alpha^{2}$, see Sec. IV A 1.

\begin{tabular}{lcccccc}
\hline \hline Method & Options & Basis set & $X_{2} 1$ & $a 2$ & $b 0^{+}$ & $\alpha^{2}$ \\
\hline Expt. & & & $\mathbf{1 2 3 4}$ & & $\mathbf{8 7 8 5}$ & \\
GASCI & $12 \mathrm{e}$ & $\mathrm{TZ}$ & 1131 & 4686 & 8933 & 0.827 \\
& $32 \mathrm{e}$ & $\mathrm{TZ}$ & 1200 & 4667 & 9000 & 0.834 \\
FSCC & dianion & $\mathrm{TZ}$ & 1067 & 5510 & 11943 & 0.784 \\
& neutral & $\mathrm{TZ}$ & 1100 & 6195 & $\ldots$ & 1 \\
& dianion & $\mathrm{QZ}$ & 1188 & 5554 & 12218 & 0.794 \\
\multirow{4}{*}{ IHFSCC } & {$[\mathrm{a}] /$ dianion spinors } & $\mathrm{QZ}$ & 1323 & 5278 & 11225 & 0.812 \\
& {$[\mathrm{a}] /$ neutral spinors } & $\mathrm{QZ}$ & 1152 & 4372 & 9166 & 0.827 \\
& {$[\mathrm{~b}] /$ neutral spinors } & $\mathrm{QZ}$ & 1239 & 4305 & 8523 & 0.842 \\
\hline \hline
\end{tabular}

tween the results with 12 and 32 correlated electrons shows that the correlation of the $(n-1) d$ sub-shell brings an important contribution: it increases the energy of the first excited $X_{2} 1$ state by more than $100 \mathrm{~cm}^{-1}$. The effect on the other states is not as large. With GASCI, when the calculation is performed with only one hole allowed in the $(n-1) d$ sub shell, one obtains similar results; it shows that the largest contribution from this sub-shell is polarization. The use of basis sets of QZ quality plays an important role as well: it improves the results by about $80 \mathrm{~cm}^{-1}$ compared to TZ basis sets. The Gaunt interaction contribution is about $-20 \mathrm{~cm}^{-1}$. By adding the contributions due to the Gaunt operator to the $4 \mathrm{c}-\mathrm{GASCI} / 32 / \mathrm{QZ}$ result, the final result would be about $1983 \mathrm{~cm}^{-1}$, almost spot on the experimental value.

With FSCC, when the spinors optimized for the dication are used in a dication scheme, the value of the ZFS is largely underestimated. This can perhaps be understood by the fact that at the SCF level, the $\pi^{*}$ orbitals are virtual in the SCF procedure. Their poor description at the HF level is not improved by the correlation treatment, whereas a dianion FSCC approach based on dianion SCF orbitals clearly yields a better ZFS value. Considering next the results obtained with the neutral-molecule FSCC scheme, we observe that the energy of the $X_{2} 1$ root is in even closer agreement with experiment, but in this case, the $X_{1} 0^{+}$state is monodeterminantal and the $b 0^{+}$cannot be calculated. The energy of the $b 0^{+}$state is, on the other hand, much too high compared to the experimental value, about 1000 and $3000 \mathrm{~cm}^{-1}$ with spinors of the dication and dianion, respectively.

IHFSCC results have been added in the revised version of the manuscript. A comparison of the IHFSCC using the [a] scheme and dianon MOs with its FSCC counterpart reveals a sizeable importance of $\pi^{2} \rightarrow \pi^{* 2}$ excitations on all excitation energies and on the composition of the wave function in the model space. The use of non-canonical neutral molecule SCF orbitals in the dianion IHFSCC [a] framework yields a significant improvement of the description of the $X_{1} 0^{+}, a 2$, and $b 0^{+}$states for both $\mathrm{Te}_{2}$ and TeSe while it slightly enlarges (reduces) the ZFS in $\mathrm{Te}_{2}$ (TeSe). These findings support the 
TABLE IV. First excited states energies (in $\mathrm{cm}^{-1}$ ) of $\mathrm{Te}_{2}$ calculated with two-step methods. $V$ is the SOC matrix element between ${ }^{3} \Sigma_{g}^{-}$and ${ }^{1} \Sigma_{g}^{+}$states.

\begin{tabular}{|c|c|c|c|c|c|c|c|c|c|}
\hline \multirow[b]{2}{*}{ Method } & \multirow[b]{2}{*}{ CAS } & \multirow[b]{2}{*}{ Rel. } & \multirow[b]{2}{*}{ Basis set } & \multicolumn{2}{|c|}{ SOF } & \multicolumn{3}{|c|}{ SOC } & \multirow[b]{2}{*}{$V$} \\
\hline & & & & ${ }^{1} \Delta_{g}$ & ${ }^{1} \Sigma_{g}^{+}$ & $X_{2} 1_{g}$ & $a 2_{g}$ & $b 0_{g}^{+}$ & \\
\hline Expt. & & & & & & 1975 & & 9600 & \\
\hline \multirow[t]{9}{*}{ SO-CASSCF } & $\operatorname{CAS}(2,2)$ & DKH & DZP & 5390 & 10784 & 638 & 6025 & 12062 & 2701 \\
\hline & $\operatorname{CAS}(2,2)$ & DKH & $\mathrm{TZP}$ & 5188 & 10740 & 652 & 5800 & 12046 & 2726 \\
\hline & $\operatorname{CAS}(2,2)$ & DKH & QZP & 5118 & 10722 & 651 & 5769 & 12056 & 2725 \\
\hline & $\operatorname{CAS}(6,4)$ & DKH & DZP & 2900 & 5397 & 1856 & 4755 & 9104 & 3665 \\
\hline & $\operatorname{CAS}(6,4)$ & DKH & $\mathrm{TZP}$ & 2947 & 5555 & 1860 & 4811 & 9271 & 3710 \\
\hline & $\operatorname{CAS}(6,4)$ & DKH & QZP & 2956 & 5577 & 1857 & 4815 & 9287 & 3712 \\
\hline & $\operatorname{CAS}(8,6)$ & DKH & DZP & 2797 & 5947 & 1684 & 4244 & 9052 & 3774 \\
\hline & $\operatorname{CAS}(8,6)$ & DKH & $\mathrm{TZP}$ & 2716 & 6009 & 1700 & 4180 & 9153 & 3807 \\
\hline & $\operatorname{CAS}(8,6)$ & DKH & QZP & 2725 & 5994 & 1713 & 4171 & 9164 & 3821 \\
\hline \multirow[t]{9}{*}{ SO-CASPT2 } & $\operatorname{CAS}(2,2)$ & DKH & DZP & 3654 & 7128 & 908 & 4836 & 8944 & 2701 \\
\hline & $\operatorname{CAS}(2,2)$ & DKH & $\mathrm{TZP}$ & 3124 & 6484 & 994 & 4170 & 8473 & 2726 \\
\hline & $\operatorname{CAS}(2,2)$ & DKH & QZP & 2905 & 6171 & 1031 & 3985 & 8234 & 2725 \\
\hline & $\operatorname{CAS}(6,4)$ & DKH & DZP & 3253 & 6139 & 1722 & 5010 & 9570 & 3671 \\
\hline & $\operatorname{CAS}(6,4)$ & DKH & $\mathrm{TZP}$ & 2753 & 5664 & 1859 & 4610 & 9369 & 3732 \\
\hline & $\operatorname{CAS}(6,4)$ & DKH & QZP & 2567 & 5500 & 1893 & 4512 & 9275 & 3734 \\
\hline & $\operatorname{CAS}(8,6)$ & DKH & DZP & 3440 & 6016 & 1651 & 4829 & 9041 & 3778 \\
\hline & $\operatorname{CAS}(8,6)$ & DKH & $\mathrm{TZP}$ & 3025 & 5588 & 1753 & 4540 & 8831 & 3818 \\
\hline & $\operatorname{CAS}(8,6)$ & DKH & QZP & 2838 & 5430 & 1793 & 4464 & 8756 & 3835 \\
\hline \multirow[t]{2}{*}{ SO-CAS+DDCI1 } & $\operatorname{CAS}(6,4)$ & DKH & $\mathrm{TZP}$ & 3194 & 6145 & 1717 & 4911 & 9580 & 3674 \\
\hline & $\operatorname{CAS}(6,4)$ & ZORA & $\mathrm{TZP}$ & 3195 & 6147 & 1628 & 4823 & 9404 & 3558 \\
\hline \multirow[t]{2}{*}{ SO-CAS+DDCI-2 } & $\operatorname{CAS}(6,4)$ & DKH & $\mathrm{TZP}$ & 3679 & 6575 & 1645 & 5324 & 9865 & 3677 \\
\hline & $\operatorname{CAS}(6,4)$ & ZORA & $\mathrm{TZP}$ & 3679 & 6577 & 1559 & 5238 & 9695 & 3580 \\
\hline \multirow[t]{2}{*}{ SO-CAS+DDCI-3 } & $\operatorname{CAS}(6,4)$ & DKH & $\mathrm{TZP}$ & 3356 & 5820 & 1794 & 5151 & 9409 & 3696 \\
\hline & $\operatorname{CAS}(6,4)$ & ZORA & $\mathrm{TZP}$ & 3357 & 5821 & 1703 & 5060 & 9228 & 3580 \\
\hline \multirow[t]{2}{*}{$\mathrm{SO}-\mathrm{CAS}+\mathrm{SD}$} & $\operatorname{CAS}(6,4)$ & DKH & $\mathrm{TZP}$ & 3219 & 5782 & 1790 & 5010 & 9365 & 3682 \\
\hline & $\operatorname{CAS}(6,4)$ & ZORA & $\mathrm{TZP}$ & 3220 & 5788 & 1699 & 4919 & 9186 & 3566 \\
\hline
\end{tabular}

hypothesis by one of the referees that our original dianion orbital dianion FSCC scheme possibly lacks significant wave function relaxation effects. Finally, the increase of the size of the total active space within the IHFSCC[b] scheme further improves the overall performance of the multi-reference coupled cluster approach, in particular, with respect to the ground $X_{1} 0^{+}$and $b 0^{+}$state description. The value of $\alpha$ obtained with this approach is very close to the GASCI one and the excitation energies of $b 0^{+}$state are very close to experimental ones: it shows that the bad description of this state by FSCC method is essentially due to the lack of decontraction of the wave function in the model space. Interestingly, the IHFSCC $[b]$ approach yields for TeSe a perfect agreement of the ZFS with experiment, whereas it exhibits for $\mathrm{Te}_{2}$ the largest deviation from experiment among all tested dianion (IH)FSCC models.

To conclude, the ZFS is close to the experimental value, $20 \mathrm{~cm}^{-1}$ below using FSCC and about $10 \mathrm{~cm}^{-1}$ over using GASCI, while the IHFSCC[b] approach tends to slightly overshoot with regard to the ZFS $\left(\mathrm{Te}_{2}\right)$, but more importantly this scheme provides a consistent description of all four electronic states of present interest. The correlation of only 12 electrons is insufficient, the inclusion of the correlation involving the $(n-1) d$ shell is necessary, at least for polarization, and basis sets of QZ quality have to be used. GASCI calculations are expensive and have only been carried out for the $\mathrm{SeO}$, $\mathrm{TeO}, \mathrm{TeSe}$, and $\mathrm{Te}_{2}$. For these molecules, we have correlated 22 electrons in the case of the oxides, and 32 electrons for the other two using the above-described $\mathrm{TZ}$ basis sets and the $\mathrm{X} 2 \mathrm{C}+$ Gaunt operator. The whole series has been described by IHFSCC calculations within the dianion scheme, the large total active space ([b]), correlating 32 electrons, using average-of-configuration spinors of the neutral molecule $X Y$, and using the $\mathrm{QZ}$ basis sets. Results for the whole series obtained by FSCC calculations within the dianion scheme using dianion spinors and QZ basis sets are given as supplementary material. ${ }^{69}$

\section{Two-step SOC methods}

Results are summarized in Tables IV and V. The SOC matrix element between $X^{3} \Sigma^{-}$and $b^{1} \Sigma^{+}$states is given: it is denoted $V$; according to the model proposed in Sec. II and Eq. (30), it is the SOC splitting of the $\pi^{*}$ spinors $\Delta \epsilon$. This matrix element plays a key role in the calculation of the ZFS. The energy of the $X_{2} 1$ state calculated at the CASSCF level with CAS $(2,2)$ is too small while the value shifts to about the right order of magnitude once the $\pi$ orbitals are included in the active space. This is due to the large non-dynamical correlation of the bond as was also deserved in the one-step calculations: the natural population of the anti-bonding $\pi^{*}$ orbital at the CASSCF level is about 0.9. Although this correlation is partly recovered at the CASPT2 level, the minimal CAS $(2,2)$ is not accurate enough; the energy of $b^{1} \Sigma^{+}$is too high and the coupling $V$ is too small. In $\mathrm{Te}_{2}$, using CAS(6,4), only excited 
TABLE V. First excited states energies (in $\mathrm{cm}^{-1}$ ) of TeSe calculated with two-step methods. For $V$, see Table IV.

\begin{tabular}{|c|c|c|c|c|c|c|c|c|c|}
\hline \multirow[b]{2}{*}{ Method } & \multirow[b]{2}{*}{ CAS } & \multirow[b]{2}{*}{ Rel. } & \multirow[b]{2}{*}{ Basis set } & \multicolumn{2}{|c|}{ SOF } & \multicolumn{3}{|c|}{ SOC } & \multirow[b]{2}{*}{$V$} \\
\hline & & & & ${ }^{1} \Delta$ & ${ }^{1} \Sigma^{+}$ & $X_{2} 1$ & $a 2$ & $b 0^{+}$ & \\
\hline Expt. & & & & & & 1234 & & 8785 & \\
\hline \multirow{9}{*}{ SO-CASSCF } & $\operatorname{CAS}(2,2)$ & DKH & DZP & 5677 & 11469 & 374 & 6051 & 12218 & 2105 \\
\hline & $\operatorname{CAS}(2,2)$ & DKH & $\mathrm{TZP}$ & 5575 & 12325 & 382 & 5955 & 12325 & 2137 \\
\hline & $\operatorname{CAS}(2,2)$ & DKH & QZP & 5562 & 11588 & 385 & 5949 & 12359 & 2147 \\
\hline & $\operatorname{CAS}(6,4)$ & DKH & DZP & 3280 & 6180 & 968 & 4226 & 8207 & 2699 \\
\hline & $\operatorname{CAS}(6,4)$ & DKH & $\mathrm{TZP}$ & 3338 & 6333 & 970 & 4284 & 8366 & 2731 \\
\hline & $\operatorname{CAS}(6,4)$ & DKH & QZP & 3336 & 6340 & 971 & 4284 & 8377 & 2735 \\
\hline & $\operatorname{CAS}(8,6)$ & DKH & DZP & 2980 & 6117 & 1021 & 3868 & 8086 & 2812 \\
\hline & $\operatorname{CAS}(8,6)$ & DKH & TZP & 2948 & 6156 & 1034 & 3848 & 8151 & 2833 \\
\hline & $\operatorname{CAS}(8,6)$ & DKH & QZP & 2909 & 6120 & 1045 & 3823 & 8139 & 2844 \\
\hline \multirow[t]{9}{*}{ SO-CASPT2 } & $\operatorname{CAS}(2,2)$ & DKH & $\mathrm{DZP}$ & 3872 & 7394 & 557 & 4430 & 9516 & 2105 \\
\hline & $\operatorname{CAS}(2,2)$ & DKH & $\mathrm{TZP}$ & 3175 & 6691 & 624 & 3800 & 7940 & 2137 \\
\hline & $\operatorname{CAS}(2,2)$ & DKH & QZP & 2947 & 6388 & 655 & 3603 & 7692 & 2147 \\
\hline & $\operatorname{CAS}(6,4)$ & DKH & DZP & 3399 & 6524 & 994 & 4380 & 8550 & 2758 \\
\hline & $\operatorname{CAS}(6,4)$ & DKH & $\mathrm{TZP}$ & 2967 & 6089 & 1064 & 4021 & 8258 & 2786 \\
\hline & $\operatorname{CAS}(6,4)$ & DKH & QZP & 2846 & 5918 & 1079 & 3914 & 8114 & 2755 \\
\hline & $\operatorname{CAS}(8,6)$ & DKH & DZP & 3567 & 6501 & 996 & 4429 & 8402 & 2856 \\
\hline & $\operatorname{CAS}(8,6)$ & DKH & TZP & 3228 & 6144 & 1053 & 4147 & 8162 & 2875 \\
\hline & $\operatorname{CAS}(8,6)$ & DKH & QZP & 3123 & 5998 & 1073 & 4063 & 8058 & 2879 \\
\hline \multirow[t]{2}{*}{ SO-CAS+DDCI1 } & $\operatorname{CAS}(6,4)$ & DKH & $\mathrm{TZP}$ & 3751 & 7146 & 883 & 4616 & 8966 & 2573 \\
\hline & $\operatorname{CAS}(6,4)$ & ZORA & $\mathrm{TZP}$ & 3752 & 7147 & 857 & 4589 & 8907 & 2653 \\
\hline \multirow{2}{*}{ SO-CAS+DDCI-2 } & $\operatorname{CAS}(6,4)$ & DKH & $\mathrm{TZP}$ & 4060 & 7393 & 884 & 4931 & 9188 & 2719 \\
\hline & $\operatorname{CAS}(6,4)$ & ZORA & TZP & 4060 & 7394 & 859 & 4906 & 9135 & 2674 \\
\hline \multirow[t]{2}{*}{ SO-CAS+DDCI-3 } & $\operatorname{CAS}(6,4)$ & DKH & $\mathrm{TZP}$ & 3790 & 6532 & 950 & 4727 & 8472 & 2691 \\
\hline & $\operatorname{CAS}(6,4)$ & ZORA & $\mathrm{TZP}$ & 3791 & 6534 & 926 & 4703 & 8421 & 2649 \\
\hline \multirow[t]{2}{*}{$\mathrm{SO}-\mathrm{CAS}+\mathrm{SD}$} & $\operatorname{CAS}(6,4)$ & DKH & $\mathrm{TZP}$ & 3680 & 6586 & 931 & 4598 & 8494 & 2676 \\
\hline & $\operatorname{CAS}(6,4)$ & ZORA & TZP & 3686 & 6596 & 906 & 4577 & 8447 & 2633 \\
\hline
\end{tabular}

states with double $\pi^{2} \rightarrow \pi^{* 2}$ excitations couple by SOC with the low lying states of configuration $\left(\pi^{*}\right)^{2}$. In TeSe, there is no inversion center and states with monoexcitations couple as well. The improvement of the ZFS value from $\operatorname{CAS}(2,2)$ to $\mathrm{CAS}(6,4)$ is not due to the presence of more states in the state interaction step but to the better description of the states arising from the $\left(\pi^{*}\right)^{2}$ configuration; both $V$ and $K$ are affected and Eq. (29) with $V$ and $K$ obtained with $\operatorname{CAS}(6,4)$ leads to the correct value of the ZFS. In $\operatorname{CAS}(8,6)$, which, in addition, comprises the $\sigma$ and $\sigma^{*}$ orbitals and the corresponding electrons, the monoexcited states $\sigma \rightarrow \pi^{*}$ and $\pi \rightarrow \sigma^{*}$ are taken into account and couple by SOC to the two ground $X$ states. This increase of the active space worsens the results. In this case, the states of $\Sigma$ symmetry play a differential role. The effect is quite important for $\mathrm{Te}_{2}\left(100 \mathrm{~cm}^{-1}\right)$ while less significant for TeSe. The effect of the size of the active space illustrates the main drawback of the two-step methods: increasing the quality of the calculation does not systematically improve the quality of the results. This already has been discussed for the calculation of $g$ factors. ${ }^{63}$

In contrast to the one-step methods discussed above, the use of basis sets of QZ quality improves only slightly the results with respect to $\mathrm{TZ}$ quality. As soon as the $\pi^{*}$ are included in the CAS, that is, from $\operatorname{CAS}(6,4)$ and beyond, the effect of correlation is not very important. The CASSCF results are already reasonably converged; neither the energy of $b^{1} \Sigma^{+}$state nor the coupling element $V$ vary significantly with the additional dynamical correlation. Comparing DDCIn calculations, results are converged at the DDCI3 level showing that the 2 holes- 2 particles contributions do not play any role. This is generally the case for the calculation of vertical excitations. ${ }^{64}$ The SO-CASSCF results are similar with the MOLCAS and ORCA codes using the same quality for the basis set and the same active space although basis sets and the SOC mean-field integrals are not identical. However, CASSDCI results are lower than the SO-CASPT2 ones using both CAS $(6,4)$. This is disappointing since the SO-DDCIn approach should be more accurate in the sense that the SOC state interaction is calculated using the whole DDCI wavefunctions, while in the case of SO-CASPT2, CASSCF wavefunctions are used and the energy matrix is dressed with 2 nd order energies. The results obtained with ZORA differ from those obtained with DKH due to the SOC step; $V$ is smaller with the former and consequently the ZFS.

The whole series will be described by the SOCAS $(8,6)$ PT2 with QZP basis set and SO-DDCI3 with TZP basis sets.

\section{B. Description of the whole series}

Results obtained with the four methods are summarized in Table VI and are compared to available experimental values and previous DFT calculations (see Table VII). Comparisons between calculated and experimental values are plotted 
TABLE VI. Calculated vertical excitations energies (in $\mathrm{cm}^{-1}$ ) in comparison with experimental data. For $\alpha^{2}$, see Sec. I and for $V$, see Table IV.

\begin{tabular}{|c|c|c|c|c|c|c|c|}
\hline & & $R(\AA)^{\mathrm{a}}$ & Expt. & GASCI & IHFSCC & SO-DDCI3 & SO-CASPT2 \\
\hline \multirow[t]{5}{*}{$\mathrm{S}_{2}$} & $X_{2} 1$ & 1.889 & $23.5^{\mathrm{b}}$ & & 25 & 18 & 19 \\
\hline & $a 2$ & & $5730^{c}$ & & 4637 & 4760 & 4161 \\
\hline & $b 0^{+}$ & & $7960^{c}$ & & 8612 & 8156 & 7758 \\
\hline & $\alpha^{2}$ & & & & 0.551 & & \\
\hline & $V$ & & & & & 384 & 387 \\
\hline \multirow[t]{5}{*}{$\mathrm{SeO}$} & $X_{2} 1$ & 1.648 & $166^{\mathrm{a}}-182^{\mathrm{d}}$ & 147 & 137 & 112 & 138 \\
\hline & $a 2$ & & $\approx 5300$ & 5614 & 5115 & 5533 & 4782 \\
\hline & $b 0^{+}$ & & $9677^{\mathrm{d}}$ & 10261 & 9166 & 9586 & 9211 \\
\hline & $\alpha^{2}$ & & & 0.618 & 0.624 & & \\
\hline & $V$ & & & & & 1062 & 1143 \\
\hline \multirow[t]{5}{*}{$\mathrm{SeS}$} & $X_{2} 1$ & 2.037 & $205^{\mathrm{a}}-207^{\mathrm{d}}$ & & 192 & 135 & 165 \\
\hline & $a 2$ & & & & 4240 & 4494 & 3930 \\
\hline & $b 0^{+}$ & & $7587^{\mathrm{d}}$ & & 7950 & 7810 & 7513 \\
\hline & $\alpha^{2}$ & & & & 0.651 & & \\
\hline & $V$ & & & & & 1044 & 1129 \\
\hline \multirow[t]{5}{*}{$\mathrm{Se}_{2}$} & $X_{2} 1$ & 2.166 & $510^{\mathrm{a}}$ & & 574 & 394 & 446 \\
\hline & $a 2$ & & & & 4242 & 4293 & 3470 \\
\hline & $b 0^{+}$ & & $7417^{\mathrm{d}}-7936^{\mathrm{e}}-7958^{\mathrm{f}}$ & & 7939 & 7731 & 7463 \\
\hline & $\alpha^{2}$ & & & & 0.744 & & \\
\hline & $V$ & & & & & 1701 & 1809 \\
\hline \multirow[t]{5}{*}{$\mathrm{TeO}$} & $X_{2} 1$ & 1.825 & $679^{\mathrm{g}}$ & 670 & 468 & 517 & 635 \\
\hline & $a 2$ & & & 5543 & 4526 & 5366 & 4427 \\
\hline & $b 0^{+}$ & & $9930^{\mathrm{h}}$ & 10540 & 9019 & 9465 & 9127 \\
\hline & $\alpha^{2}$ & & & 0.743 & 0.744 & & 2400 \\
\hline & $V$ & & & & & 2691 & \\
\hline \multirow[t]{5}{*}{$\mathrm{TeS}$} & $X_{2} 1$ & 2.230 & $836^{\mathrm{i}}$ & & 619 & 590 & 675 \\
\hline & $a 2$ & & & & 3993 & 4594 & 3975 \\
\hline & $b 0^{+}$ & & $8446^{\mathrm{i}}$ & & 8177 & 8220 & 7912 \\
\hline & $\alpha^{2}$ & & & & 0.775 & & \\
\hline & $V$ & & & & & 2198 & 2307 \\
\hline \multirow[t]{5}{*}{$\mathrm{TeSe}$} & $X_{2} 1$ & 2.372 & $1233^{\mathrm{j}}-1241^{\mathrm{k}}$ & 1200 & 1238 & 950 & 1073 \\
\hline & $a 2$ & & $7752^{j}$ & 4667 & 4305 & 4727 & 4063 \\
\hline & $b 0^{+}$ & & $8785^{\mathrm{k}}$ & 9000 & 8523 & 8472 & 8058 \\
\hline & $\alpha^{2}$ & & & 0.834 & 0.842 & & \\
\hline & $V$ & & & & & 2691 & 2879 \\
\hline \multirow[t]{5}{*}{$\mathrm{Te}_{2}$} & $X_{2} 1$ & 2.557 & $1975^{a}$ & 1929 & 2178 & 1794 & 1793 \\
\hline & $a 2$ & & & 5037 & 5029 & 5151 & 4464 \\
\hline & $b 0^{+}$ & & $9600^{1}$ & 9717 & 9724 & 9409 & 8756 \\
\hline & $\alpha^{2}$ & & & 0.889 & 0.898 & & \\
\hline & $V$ & & & & & 3696 & 3825 \\
\hline \multirow[t]{5}{*}{$\mathrm{Po}_{2}$} & $X_{2} 1$ & $2.795^{\mathrm{m}}$ & & & 7470 & & 6903 \\
\hline & $a 2$ & & & & 9665 & & 7619 \\
\hline & $b 0^{+}$ & & & & 16864 & & 16271 \\
\hline & $\alpha^{2}$ & & & & 0.974 & & \\
\hline & $V$ & & & & & & 11370 \\
\hline
\end{tabular}

${ }^{\mathrm{a} F r o m ~ R e f . ~} 51$.

${ }^{\mathrm{b}}$ From Refs. 65 and 66.

${ }^{\mathrm{c}}$ From Ref. 54.

${ }^{\mathrm{d}}$ From Ref. 55.

${ }^{\mathrm{e}}$ From Ref. 56.

${ }^{\mathrm{f}}$ From Ref. 60.

${ }^{\mathrm{g}}$ From Ref. 67.

${ }^{\text {h}}$ From Ref. 57.

${ }^{\mathrm{i}}$ From Ref. 57.

${ }^{\mathrm{j}}$ From Ref. 59.

${ }^{\mathrm{k}}$ From Ref. 58.

${ }^{1}$ From Ref. 61.

${ }^{\mathrm{m}}$ Optimized in this work. 


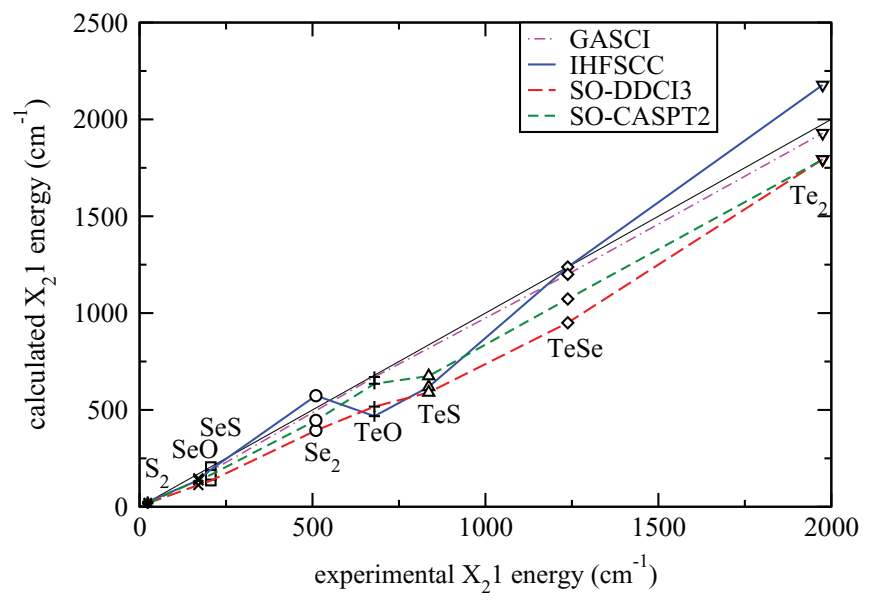

FIG. 1. Comparison of calculated and experimental values of the energy of $X_{2} 1$ state.

in Figure 1 for the $X_{2} 1$ state and Figure 2 for the $b 0^{+}$ state. Concerning the $X_{2} 1$ state, GASCI, IHFSCC, SO-DDCI, and SO-CASPT2 give the experimental values to within $6 \%$, $14 \%, 25 \%$, and $15 \%$, respectively. SO-DDCI systematically overestimates the excitation energy of the $b^{1} \Sigma^{+}$states and underestimates the coupling $V$. Turning to the $b 0^{+}$state, these methods are able to reproduce the experimental values to within $4 \%, 5 \%, 3 \%$, and $5 \%$, respectively. There are three experimental studies of the $b 0_{g}^{+}$state of $\mathrm{Se}_{2}$ : the first one using chemiluminescence gives $7417 \mathrm{~cm}^{-1}$ for the $b 0_{g}^{+} \rightarrow X_{1} 0_{g}^{+}$transition. $^{55}$ The next study, using high resolution Fourier transform fluorescence spectroscopy, gives $7958 \mathrm{~cm}^{-1}$ for the same transition. ${ }^{60}$ New chemiluminescence experiments reassign the $7417 \mathrm{~cm}^{-1}$ to the $b 0_{g}^{+}$ $\rightarrow X_{2} 1_{g}$ transition which is magnetic dipole allowed, giving $7960 \mathrm{~cm}^{-1}$ for the $b 0_{g}^{+} \rightarrow X_{1} 0_{g}^{+}$transition. ${ }^{56}$ However, the calculated values for the $b 0^{+}$state by the three different methods give smaller values for $\mathrm{Se}_{2}$ than for $\mathrm{SeS}$. The experimental value of $7417 \mathrm{~cm}^{-1}$ for this state is more in line with our calculations.

The FSCC results are given as supplementary material. We did not manage to correctly describe molecules contain-

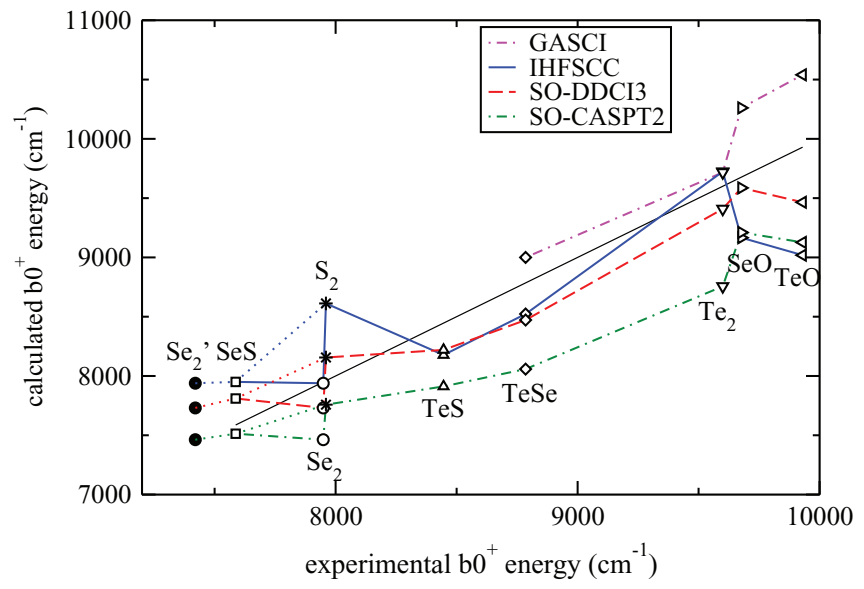

FIG. 2. Comparison of calculated and experimental values of the energy of $b 0^{+}$state. $\mathrm{Se}_{2}$ (white circles) and $\mathrm{Se}_{2}^{\prime}$ (black circles) correspond to experimental values of Refs. 55 and 60, respectively.
TABLE VII. ZFS calculated with DFT (in $\mathrm{cm}^{-1}$ ).

\begin{tabular}{lrc}
\hline \hline & CP-B3LYP $^{\mathrm{a}}$ & 2c-BP98 $^{\mathrm{b}}$ \\
\hline $\mathrm{S}_{2}$ & 10 & 20 \\
$\mathrm{SeO}$ & 157 & 70 \\
$\mathrm{SeS}$ & 84 & 186 \\
$\mathrm{Se}$ & 224 & 498 \\
$\mathrm{TeO}$ & 368 & \\
$\mathrm{TeS}$ & 402 & \\
$\mathrm{TeSe}$ & 638 & \\
$\mathrm{Te}_{2}$ & 1115 & \\
\hline
\end{tabular}

${ }^{a}$ From Ref. 20.

${ }^{\mathrm{b}}$ From Ref. 18

ing an oxygen atom given the range of quality for the other molecules: the results are strongly dependent on the starting orbitals and provide another indication of the importance of explicitly taking into account $\pi \rightarrow \pi^{*}$ interactions which has already been discussed in Sec. IV A. While the ZFS is very good for the molecules without oxygen, the energy of the $b 0^{+}$ state is largely overestimated in the whole series.

The $\Delta \varepsilon$ and $K$ parameters of Sec. II have been extracted by a mean-square fit using the energies of the four states arising from the $\left(\pi^{*}\right)^{2}$ configuration and are given in Table VIII (corresponding FSCC values are given in the supplementary material). The energy gap between $\pi_{3 / 2}^{*}$ and $\pi_{1 / 2}^{*}$, corresponding to $\Delta \varepsilon$, can be directly obtained from one-step SOC calculations: The orbital energy difference $\varepsilon_{3 / 2}-\varepsilon_{1 / 2}$ has been extracted from average-of-configuration Hartree-Fock calculations with one open-shell containing 2 electrons in 4 spinors $\left(\pi_{1 / 2}^{*}, \pi_{3 / 2}^{*}\right)$. It is systematically larger than $V$ by some hundreds of $\mathrm{cm}^{-1}$ : This difference is rather constant and can therefore not be due to $\sigma / \pi$ hybridization by SOC. $K$ decreases in the series since the orbitals become more and more diffuse. The values of $K$ extracted from IHFSCC and SOCASPT2 calculations are quite similar, whereas the GASCI ones are slightly larger and the FSCC values vary significantly. The parameters obtained with the FSCC and IHFSCC methods are very different since the effective matrices corresponding to matrix (24) are very different. As seen in Figure $3, \Delta \varepsilon$ varies almost linearly with $K$ except for

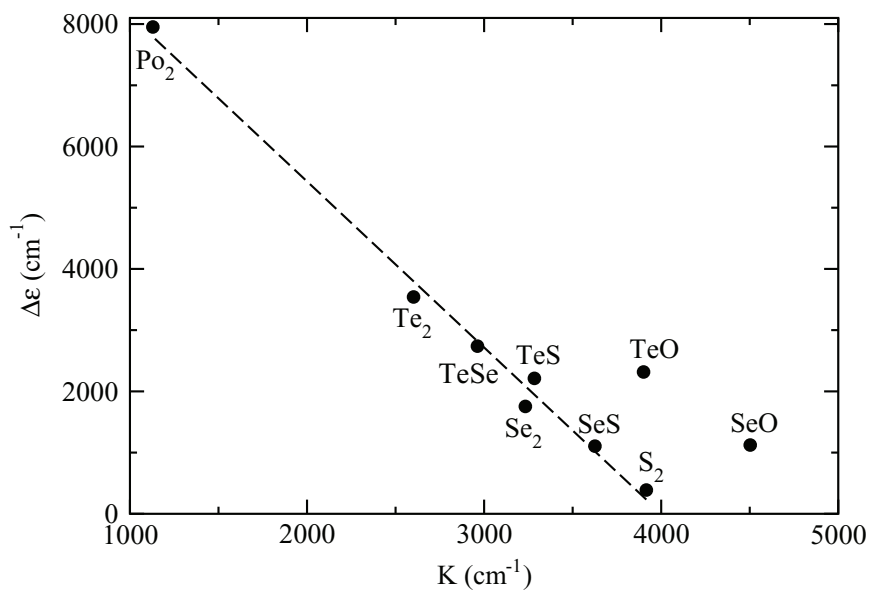

FIG. 3. $\Delta \varepsilon$ vs $K$ from SO-CASPT2 calculations. 
TABLE VIII. Parameters $\Delta \varepsilon$ and $K\left(\right.$ in $\left.\mathrm{cm}^{-1}\right)$.

\begin{tabular}{|c|c|c|c|c|c|c|c|c|c|c|}
\hline & \multicolumn{3}{|c|}{ GASCI } & \multicolumn{3}{|c|}{ IHFSCC } & \multicolumn{3}{|c|}{ SO-CASPT2 } & \multirow[b]{2}{*}{$\zeta^{\mathrm{c}}$} \\
\hline & $\Delta \varepsilon^{\mathrm{a}}$ & $K^{\mathrm{a}}$ & $\varepsilon_{3 / 2}-\varepsilon_{1 / 2}{ }^{\mathrm{b}}$ & $\Delta \varepsilon^{\mathrm{a}}$ & $K^{\mathrm{a}}$ & $\varepsilon_{3 / 2}-\varepsilon_{1 / 2}{ }^{b}$ & $\Delta \varepsilon^{\mathrm{a}}$ & $K^{\mathrm{a}}$ & $V$ & \\
\hline $\mathrm{S}_{2}$ & & & & 466 & 4347 & 501 & 386 & 3916 & 387 & 386 \\
\hline $\mathrm{SeO}$ & 1230 & 5080 & 1546 & 1125 & 4552 & 1556 & 1123 & 4503 & 1143 & \\
\hline $\mathrm{SeS}$ & & & & 1228 & 3836 & 1437 & 1106 & 3626 & 1129 & \\
\hline $\mathrm{Se}_{2}$ & & & & 2071 & 3450 & 2197 & 1755 & 3233 & 1809 & 1803 \\
\hline $\mathrm{TeO}$ & 2585 & 4654 & 3241 & 2001 & 4044 & 3239 & 2315 & 3901 & 2400 & \\
\hline $\mathrm{TeS}$ & & & & 2122 & 3330 & 2891 & 2211 & 3284 & 2307 & \\
\hline $\mathrm{TeSe}$ & 2987 & 3379 & 3349 & 3006 & 3032 & 3347 & 2740 & 2962 & 2879 & \\
\hline $\mathrm{Te}_{2}$ & 3893 & 2965 & 4145 & 4072 & 2717 & 4105 & 3542 & 2602 & 3825 & 3915 \\
\hline $\mathrm{Po}_{2}$ & & & & 8594 & 1208 & 6524 & 7952 & 1129 & 11370 & 12369 \\
\hline
\end{tabular}

${ }^{a}$ Optimized by a mean-square fit using the energies of the four states arising from the $\left(\pi^{*}\right)^{2}$ configuration.

${ }^{\mathrm{b}}$ Difference between the canonical energies of $\pi_{1 / 2}^{*}$ and $\pi_{3 / 2}^{*}$ at the HF level.

${ }^{\mathrm{c}}$ From Appendix.

oxygen-containing molecules that have larger values of $K$ than the rest of the series. The energy of the $a 2$ state lies at $\sqrt{\Delta \varepsilon^{2}+K^{2}}$ above the ground state. Its energy first diminishes due to the lowering of $K$ and then increases due to the increase of $\Delta \varepsilon$.

As expected, $\Delta \varepsilon$ increases along the series. In general, we find that the $\Delta \varepsilon$ and $V$ extracted from SO-CASPT2 are quite similar, in accordance with the model of Sec. II. Exceptions are $\mathrm{Te}_{2}$, presumably due to the effect of the $\Sigma$ states, and $\mathrm{Po}_{2}$, for which the model breaks down due to secondorder SOC. For the homonuclear species we observe that $V$ is, in general, close to the atomic SOC constant $\zeta$. The model proposed in Sec. II allows us to rationalize the evolution of all the states arising from the $\pi^{* 2}$ configuration.

\section{CONCLUSIONS}

The four low-lying states of the chalcogen diatomics have been studied using wave function based methods. The first excited state, arising from the splitting of the ground state due to SOC is described with high accuracy by the GASCI method, while two-step methods and the IHFSCC method, on the other hand, have an accuracy of about $80 \%$ for these states. The third excited state $b 0^{+}$is very well described by the four considered methods. The lower spectrum of these species can be rationalized by two parameters: the SOC splitting of the $\pi^{*}$ spinors ultimately leads to a stabilized closed-shell ground state, but is opposed by the exchange energy between the same spinors.

TABLE IX. Experimental energies of the atomic states issued from the $n p^{4}$ configuration, extracted parameters, and mean square error $\left(\right.$ in $\mathrm{cm}^{-1}$ ). (See Ref. 68.)

\begin{tabular}{lrrrcccccr}
\hline \hline & ${ }^{3} P_{1}$ & ${ }^{3} P_{0}$ & ${ }^{1} D_{2}$ & ${ }^{1} S_{0}$ & \multicolumn{1}{c}{$\zeta$} & $E_{D}$ & $E_{S}$ & mse & ${ }^{2}{ }^{3} P_{0}$ \\
\hline $\mathrm{O}$ & 158 & 226 & 15867 & 33792 & 153 & 15790 & 33713 & 1 & 151 \\
$\mathrm{~S}$ & 396 & 573 & 9239 & 22181 & 386 & 9029 & 22181 & .4 & 382 \\
$\mathrm{Se}$ & 1989 & 2534 & 9576 & 22444 & 1803 & 8328 & 21036 & 4 & 1689 \\
$\mathrm{Te}$ & 4751 & 4707 & 10559 & 23199 & 3915 & 7036 & 18591 & 21 & 3138 \\
$\mathrm{Po}$ & 16831 & 7514 & 21679 & 42718 & 12369 & 6605 & 16608 & 9 & 5009 \\
\hline \hline
\end{tabular}

\section{ACKNOWLEDGMENTS}

This work has been supported by the ANR-09-BLAN0195 TEMAMA. S.K. gratefully acknowledges postdoctoral research grants from l'Université de Strasbourg (UdS) and the Natural Science Foundation (FNU) of the Danish Agency for Science, Technology, and Innovation. This work has been supported through ample computing time at the supercomputer centers of UdS and the Danish Center for Scientific Computing at SDU Odense. F.N. and D.G. gratefully acknowledge financial support of this work by the University of Bonn, the Max Planck Institut, and the SFB 813 ("Chemistry at spin centers"). The authors would like to acknowledge interesting and most helpful remarks of the referees.

\section{APPENDIX: ATOMIC SPIN-ORBIT CONSTANTS}

The atomic SOC constant $\zeta$ can be evaluated from atomic spectra. The $p^{4}$ configuration gives rise to ${ }^{3} P,{ }^{1} D$, and ${ }^{1} S$ states. The splitting of the SOF ground state ${ }^{3} P$ in ${ }^{3} P_{2},{ }^{2} P_{1}$, and ${ }^{3} P_{0}$ states by first order SOC gives a first estimate of $\zeta$. But, with increasing values of $\zeta$, the Landé rule is not fulfilled due to 2 nd order SOC with the ${ }^{1} D_{2}$ and ${ }^{1} S_{1}$ states, and for atoms heavier than Te, there even is an inversion of ${ }^{2} P_{1}$ and ${ }^{3} P_{0}$ states. A three-parameters extraction is possible using a mean square approach; in supplement to $\zeta$, the energies $E_{D}$ and $E_{S}$ of the SOF ${ }^{1} D_{2}$ and ${ }^{1} S_{1}$ states are introduced. Results are summarized in Table IX. The obtained value of $\zeta$ is compared to the value obtained from the 1st order splitting of ${ }^{3} P$. The discrepancy becomes large for Se and onwards.

${ }^{1}$ D. Gatteschi, R. Sessoli, and J. Villain, Molecular Nanomagnets (Oxford University Press, Oxford, 2006).

${ }^{2}$ H. A. Kramers, Z. Phys. 53, 422 (1929).

${ }^{3}$ J. H. Van Vleck and W. G. Penney, Philos. Mag. 17, 961 (1934).

${ }^{4}$ R. Schlapp and W. G. Penney, Phys. Rev. 42, 666 (1932).

${ }^{5}$ M. H. L. Pryce, Phys. Rev. 80, 1107 (1950).

${ }^{6}$ M. Tinkham and M. W. P. Strandberg, Phys. Rev. 97, 937 (1955).

${ }^{7}$ H. F. Hameka, J. Chem. Phys. 31, 315 (1959).

${ }^{8}$ M. Godfrey, C. W. Kern, and M. Karplus, J. Chem. Phys. 44, 4459 (1966).

${ }^{9}$ Z. Havlas, J. W. Downing, and J. Michl, J. Phys. Chem. A 102, 5681 (1998).

${ }^{10}$ O. Vahtras, O. Loboda, B. Minaev, H. Ågren, and K. Ruud, Chem. Phys. 279, 133 (2002)

${ }^{11}$ N. Gilka, P. N. Taylor, and C. M. Marian, J. Chem. Phys. 129, 044102 (2008). 
${ }^{12}$ T. T. Petrenko, T. L. Petrenko, and V. Ya Bratus, J. Phys.: Condens. Matter 14, 12433 (2002).

${ }^{13}$ S. Sinnecker and F. Neese, J. Phys. Chem. A 110, 12267 (2006).

${ }^{14}$ F. Neese, J. Am. Chem. Soc. 128, 10213 (2006).

${ }^{15}$ M. H. L. Pryce, Proc. Phys. Soc., London, Sect. A 63, 25 (1950).

${ }^{16}$ A. Bencini, C. Benelli, and D. Gatteschi, Coord. Chem. Rev. 60, 131 (1984).

${ }^{17}$ M. R. Pederson and S. N. Khanna, Phys. Rev. B 60, 9566 (1999).

${ }^{18}$ F. Neese, J. Chem. Phys. 127, 164112 (2007).

${ }^{19}$ C. van Wüllen, J. Chem. Phys. 130, 194109 (2009).

${ }^{20}$ R. Reviakine, A. V. Arbuznikov, J.-C. Tremblay, C. Remenyi, O. L. Malkina, V. G. Malkin, and M. Kaupp, J. Chem. Phys. 125, 054110 (2006).

${ }^{21}$ Z. Havlas and J. Michl, J. Chem. Soc., Perkin Trans. 2 1999, 2299.

${ }^{22}$ O. Loboda, B. Minaev, O. Vahtras, B. Schimmelpfennig, H. Ågren, K. Ruud, and D. Jonsson, Chem. Phys. 286, 127 (2003).

${ }^{23}$ C. de Graaf and C. Sousa, Int. J. Quantum Chem. 106, 2470 (2006).

${ }^{24}$ S. Petit, G. Pilet, L. F. Luneau, D. Chibotaru, and L. Ungur, Dalton Trans. 40, 4582 (2007).

${ }^{25}$ R. Maurice, R. Bastardis, C. de Graaf, N. Suaud, T. Mallah, and N. Guihery, J. Chem. Theory Comput. 11, 2977 (2009).

${ }^{26}$ D. Ganyushin and F. Neese, J. Chem. Phys. 125, 024103 (2006).

${ }^{27}$ dirac, a relativistic $a b$ initio electronic structure program, written by T. Saue, L. Visscher, and H. J. Aa. Jensen, release DIRAC10, R. Bast, K. G. Dyall, U. Ekström, et al.

${ }^{28}$ J. Sikkema, L. Visscher, T. Saue, and M. Iliaš, J. Chem. Phys. 131, 124116 (2009).

${ }^{29}$ M. Ilias and T. Saue, J. Chem. Phys. 126, 064102 (2007).

${ }^{30}$ B. A. Hess, C. M. Marian, U. Wahlgren, and O. Gropen, Chem. Phys. Lett. 251, 365 (1996).

${ }^{31}$ B. Schimmelpfennig, L. Maron, U. Wahlgren, C. Teichteil, H. Fagerli, and O. Gropen, Chem. Phys. Lett. 286, 267 (1998).

${ }^{32}$ L. Visscher, E. Eliav, and U. Kaldor, J. Chem. Phys. 115, 9720 (2001).

${ }^{33}$ A. Landau, E. Eliav, Y. Ishikawa, and U. Kaldor, J. Chem. Phys. 113, 9905 (2000).

${ }^{34}$ A. Landau, E. Eliav, Y. Ishikawa, and U. Kaldor, J. Chem. Phys. 115, 6862 (2001).

${ }^{35}$ K. G. Dyall, Theor. Chem. Acc. 115, 441 (2006).

${ }^{36}$ T. Fleig, H. J. Aa Jensen, J. Olsen, and L. Visscher, J. Chem. Phys. 124, 104106 (2006).

${ }^{37}$ S. Knecht, H. J. Aa Jensen, and T. Fleig, J. Chem. Phys. 132, 014108 (2010).

${ }^{38}$ T. Fleig, J. Olsen, and C. M. Marian, J. Chem. Phys. 114, 4775 (2001).

${ }^{39} \mathrm{~F}$. Neese, ORCA, an ab initio, density functional, and semiempirical program package, Universität Bonn, Bonn, 2007.

${ }^{40}$ F. Neese, J. Chem. Phys. 119, 9428 (2003).

${ }^{41}$ J. Miralles, J.-P. Daudey, and R. Caballol, Chem. Phys. Lett. 198, 555 (1992).

${ }^{42}$ B. A. Hess, Phys. Rev. A 33, 3742 (1986).
${ }^{43}$ E. van Lenthe, P. E. S. Wormer, and A. van der Avoird, J. Chem. Phys. 107, 4775 (1997).

${ }^{44}$ A. Schäffer, C. Huber, and R. Ahlrichs, J. Chem. Phys. 100, 5829 (1994).

${ }^{45}$ G. Karlström, R. Lindh, P.-Å. Malmqvist, B. O. Roos, U. Ryde, V. Veryazov, P.-O. Widmark, M. Cossi, B. Schimmelpfennig, P. Neogrady, and L. Seijo, Comput. Mater. Sci. 28, 222 (2003).

${ }^{46}$ B. O. Roos, R. Lindh, P.-Å. Malmqvist, V. Veryazov, and P. O. Widmark, J. Phys. Chem. A 108, 2851 (2004).

${ }^{47}$ B. O. Roos, R. Lindh, P.-Å. Malmqvist, V. Veryazov, and P. O. Widmark, J. Phys. Chem. A 109, 6575 (2005).

${ }^{48}$ P.-Å. Malmqvist, A. Rendell, and B. O. Roos, J. Phys. Chem. 94, 5477 (1990).

${ }^{49}$ K. Andersson, P.-Å. Malmqvist, B. O. Roos, A. J. Sadlej, and K. Wolinski, J. Phys. Chem. 94, 5483 (1990).

${ }^{50}$ P.-Å. Malmqvist, B. O. Roos, and B. Schimmelpfennig, Chem. Phys. Lett. 357, 230 (2002).

${ }^{51}$ K. P. Huber and G. Herzberg, Molecular Spectra and Molecular Structure: Constants of Diatomic Molecules (Van Nostrand Reinhold, New York, 1979).

${ }^{52}$ R. F. Barrow and K. K. Yee, Acta Phys. Acad. Sci. Hung. 35, 239 (1974).

${ }^{53}$ I. Barnes, K. H. Becker, and E. H. Fink, Chem. Phys. Lett. 67, 310 (1979).

${ }^{54}$ I. Barnes, K. H. Becker, and E. H. Fink, Chem. Phys. Lett. 67, 314 (1979).

${ }^{55}$ R. Winter, I. Barnes, E. H. Fink, J. Wildt, and F. Zabel, Chem. Phys. Lett. 73, 297 (1980).

${ }^{56}$ R. Winter, I. Barnes, E. H. Fink, J. Wildt, and F. Zabel, Chem. Phys. Lett. 86, 118 (1982).

${ }^{57}$ R. Winter, I. Barnes, E. H. Fink, J. Wildt, and F. Zabel, J. Mol. Struct. 80, 75 (1982).

${ }^{58}$ R. Winter, E. H. Fink, J. Wildt, and F. Zabel, Chem. Phys. Lett. 94, 335 (1983).

${ }^{59}$ E. H. Fink, K. D. Setzer, D. A. Ramsay, M. Vervloet, and G. Z. Xu, J. Mol. Spectrosc. 136, 218 (1989).

${ }^{60}$ S. J. Prosser, R. F. Barrow, J. Vergés, C. Effantin, and J. d'Incan, J. Phys. B 13, L547 (1980).

${ }^{61}$ C. Effantin, J. D'Incan, M. T. Macpherson, and R. F. Barrow, Chem. Phys. Lett. 70, 560 (1980).

${ }^{62}$ J. Vergés, C. Effantin, O. Babaky, J. d'Incan, S. J. Prosser, and R. F. Barrow, Phys. Scr. 25, 338 (1982).

${ }^{63} \mathrm{H}$. Bolvin, ChemPhysChem 7, 1575 (2006).

${ }^{64}$ H. Bolvin, J. Phys. Chem. A 107, 5071 (2003).

${ }^{65}$ F. D. Wayne, P. B. Davies, and B. A. Thrush, Mol. Phys. 28, 989 (1974).

${ }^{66}$ H. M. Pickett and T. L. Boyd, J. Mol. Spectrosc. 75, 53 (1979).

${ }^{67}$ R. F. Barrow and M. R. Hitchings, J. Phys. B 5, L132 (1972).

${ }^{68}$ C. E. Moore, Atomic Energy Levels (National Bureau of Standards, USA, 1971).

${ }^{69}$ See supplementary material at http://dx.doi.org/10.1063/1.3636084 for the table summarizing the FSCC results. 